To Appear in ApJS

\title{
GASS: The Parkes Galactic All-Sky Survey. I. Survey Description, Goals, and Initial Data Release
}

\author{
N. M. McClure-Griffiths, ${ }^{1}$ D. J. Pisano, ${ }^{2,3}$ M. R. Calabretta, ${ }^{1}$ H. Alyson Ford,,${ }^{1,4}$ Felix J. \\ Lockman, ${ }^{2}$ L. Staveley-Smith, ${ }^{5}$ P. M. W. Kalberla, ${ }^{6}$ J. Bailin,${ }^{7}$ L. Dedes, ${ }^{6}$ S. Janowiecki,${ }^{2,8}$ B. K. \\ Gibson, ${ }^{9}$ T. Murphy, ${ }^{10,11}$ H. Nakanishi, ${ }^{12}$ K. Newton-McGee ${ }^{1,10}$
}

\begin{abstract}
The Parkes Galactic All-Sky Survey (GASS) is a survey of Galactic atomic hydrogen ( $\mathrm{H} \mathrm{I}$ ) emission in the Southern sky covering declinations $\delta \leq 1^{\circ}$ using the Parkes Radio Telescope. The survey covers $2 \pi$ steradians with an effective angular resolution of $\sim 16^{\prime}$, at a velocity resolution of $1.0 \mathrm{~km} \mathrm{~s}^{-1}$, and with an rms brightness temperature noise of $57 \mathrm{mK}$. GASS is the most sensitive, highest angular resolution survey of Galactic $\mathrm{H}$ I emission ever made in the Southern sky. In this paper we outline the survey goals, describe the observations and data analysis, and present the first-stage data release. The data product is a single cube at full resolution, not corrected for stray radiation. Spectra from the survey and other data products are publicly available online.
\end{abstract}

\footnotetext{
${ }^{1}$ Australia Telescope National Facility, CSIRO, Marsfield NSW 2122, Australia; naomi.mcclure-griffiths@csiro.au, mark.calabretta@csiro.au

${ }^{2}$ National Radio Astronomy Observatory, Green Bank, WV 24944; dpisano@nrao.edu, jlockman@nrao.edu

${ }^{3}$ present address: Department of Physics, West Virginia University, Morgantown, WV 26506

${ }^{4}$ Centre for Astrophysics and Supercomputing, Swinburne University of Technology, Hawthorn VIC 3122, Australia; alyson@astro.swin.edu.au

${ }^{5}$ School of Physics, University of Western Australia, Crawley WA 6009, Australia; lister.staveley-smith@uwa.edu

${ }^{6}$ Argelander-Institut für Astronomie, Universität Bonn, 53121 Bonn, Germany; pkalberla@astro.uni-bonn.de, ldedes@astro.uni-bonn.de

${ }^{7}$ Department of Physics and Astronomy, McMaster University, Hamilton, ON L8S 4M1, Canada; bailinj@mcmaster.ca

${ }^{8}$ Department of Astronomy, Case Western Reserve University, Cleveland, OH 44106; present address: Department of Astronomy, Indiana University Bloomington, IN 47405; sjanowie@indiana.edu

${ }^{9}$ Centre for Astrophysics, University of Central Lancashire, Preston, PR1 2HE, UK; bkgibson@uclan.ac.uk

${ }^{10}$ School of Physics, The University of Sydney, NSW 2006, Australia; tara@physics.usyd.edu.au

${ }^{11}$ School of Information Technologies, The University of Sydney, NSW 2006, Australia

${ }^{12}$ Faculty of Science, Kagoshima University, Kagoshima 890-0068, Japan; hnakanis@sci.kagoshima-u.ac.jp
} 
Subject headings: surveys - ISM: general — radio lines: ISM — galaxies: interactions — Galaxy: structure - Magellanic Clouds

\section{Introduction}

Atomic hydrogen ( $\mathrm{H} \mathrm{I}$ ) is a ubiquitous component of disk galaxies. Most readily traced by the $\lambda=21 \mathrm{~cm}$ spectral line, $\mathrm{H}$ I from the Milky Way is observed in all directions of the sky. It is possible to trace Galactic H I emission to the far side of the Milky Way, probing Galactic and interstellar processes in regions of the Galaxy that are inaccessible at many other wavelengths. As the dominant component of the interstellar medium (ISM) by number, H I allows us to trace a wide variety of Galactic processes including the impact of massive stars on the ISM (e.g., Heiles 1984; McClure-Griffiths et al. 2002); the interaction of the Galactic disk and halo (e.g., McClure-Griffiths et al. 2006; Lockman et al. 2008); the ISM life-cycle; and the formation of cold clouds (e.g., Gibson et al. 2000; Kavars et al. 2005). Since the discovery of the $\lambda=21 \mathrm{~cm}$ spectral line in 1951 by Ewen \& Purcell (1951), it has been used repeatedly, and with continuous refinements, to explore the rotation curve and map the global structure of the Galaxy (e.g., Kerr 1962; Henderson et al. 1982; Levine et al. 2006; McClure-Griffiths \& Dickev 2007).

Not long after the discovery of the $\mathrm{H}$ I spectral line, $\mathrm{H}$ I emission was found at velocities in excess of $\left|V_{L S R}\right| \gtrsim 100 \mathrm{~km} \mathrm{~s}^{-1}$ that could not be explained by Galactic rotation (Muller et al. 1963; Smith 1963). These high velocity clouds (HVCs) are now known to cover a significant fraction of the sky (Wakker \& van Woerden 1997). HVCs are observed at both positive and negative LSR velocities with magnitudes up to $\left|V_{L S R}\right| \lesssim 500 \mathrm{~km} \mathrm{~s}^{-1}$. There have been extensive searches for high velocity gas (see Wakker \& van Woerden 1997 for a review, or recent surveys by Putman et al. 2002 and Lockman et al. 2002b). It now seems certain that HVCs represent a variety of phenomena. Some HVCs may be related to a Galactic fountain (e.g., Bregman 1980); some are tidal debris, such as those connected to the Magellanic Stream (e.g., Putman et al. 2003) or other satellites

(e.g. Lockman 2003); some may be infalling intergalactic gas (e.g., Complex C; Wakker et al. 1999; Tripp et al. 2003; Lockman et al. 2008)1; and some may be associated with gas condensing from a massive, hot halo (Maller \& Bullock 2004; Sommer-Larsen 2006). Studies of the structure and distribution of high velocity gas provide critical information on the evolution of the Milky Way system.

All-sky surveys of Galactic H I with broad bandwidths and high spectral and angular resolution allow astronomers to simultaneously explore Milky Way structure, the ISM and HVCs. The most recent such survey is the Leiden-Argentine-Bonn survey (LAB; Kalberla et al. 2005), which has produced a sensitive $\left(\sigma T_{B}=70-90 \mathrm{mK}\right)$ database of the entire sky with a beam size of $\sim$ $30^{\prime}$ sampled on a $30^{\prime}$ grid, giving an effective resolution of $\sim 1^{\circ}$. However, we know from high resolution studies of targeted areas of the Galactic halo $\mathrm{H}$ I emission that there is a wealth of 
interesting structure on scales smaller than 30' (Lockman 2002; Stanimirović et al. 2006; Peek et al. 2007). For example, Green Bank Telescope (GBT) results from Lockman (2002) show that the halo of the Galaxy contains a significant population of small, cold clouds, possibly products of the Galactic fountain, that are not detectable with the angular resolution of the LAB survey. Similarly, high angular and spectral resolution studies have resolved physical and spectral structure in many HVCs, giving clues to their nature (Brüns et al. 2005; Westmeier et al. 2005). The HIPASS survey (Barnes et al. 2001, Putman et al. 2002) covered the sky at $\sim 15^{\prime}$ resolution, but was not designed for Galactic H I, and therefore did not accurately measure low velocity gas, nor have the velocity resolution needed to resolve interstellar H I lines. While high angular resolution Galactic Plane surveys (Taylor et al. 2003; McClure-Griffiths et al. 2005; Stil et al. 2006) have allowed us to carefully explore Galactic $\mathrm{H}$ I emission in the disk, they are not very sensitive $\left(\sigma T_{B} \sim 1 \mathrm{~K}\right)$ and are restricted to a few degrees around the Galactic Plane, leaving most of the volume of the Galaxy unexplored on scales less than a degree. To fully understand the nature and origin of $\mathrm{H}$ I structure in the halo and of high velocity clouds there is a need for a sensitive, unbiased high resolution survey of the entire sky.

We have made a new sensitive, fully sampled, high resolution sky survey of Galactic H I emission south of declination $\delta=1^{\circ}$, with the Parkes $64 \mathrm{~m}$ radio telescope: the Galactic AllSky Survey (GASS). It was designed with the primary goals of studying the interaction of the Milky Way disk and halo and the nature of HVCs and intermediate velocity clouds (IVCs; e.g., Kuntz \& Danly 1996). Secondary science goals include Milky Way structure and the extended structure of the Magellanic system. Here we describe the GASS project, focusing on the survey goals and techniques. In $\$ 2.1$ we describe the main design goals of the survey and the details about the observations and data reduction techniques are given in $\$ 2.2$ and $\$ 2.3$, respectively. In $\S 3$ we present the data products and discuss the limitations of this first stage of our two-stage data release. Finally, in 4 we briefly discuss some applications of the first release data and describe some initial GASS results.

\section{Observations and Data Reduction}

\subsection{Survey Design}

The parameters of the survey were chosen to match specific criteria relating to the spatial and velocity extents and velocity widths of known $\mathrm{H}$ I emission. Accurate measurement of Galactic H I at all velocities requires that the data be taken by frequency-switching, as $\mathrm{H}$ I emission, especially at $V_{L S R} \approx 0 \mathrm{~km} \mathrm{~s}^{-1}$, is always very extended. The survey had to cover at least $-400<V_{L S R}<+400$ $\mathrm{km} \mathrm{s}^{-1}$, which is the range of high-velocity H I in the Southern hemisphere (Putman et al. 2002). Throughout this work all velocities are given in the kinematic Local Standard of Rest (LSRK), also commonly referred to simply as LSR, and defined from an average of the velocities of stars in the Solar neighbourhood (Delhave 1965; Gordon 1976). The velocity resolution had to be sufficient 
to measure the narrow lines (a few $\mathrm{km} \mathrm{s}^{-1}$ ) found in HVC cores and disk H I (Haud \& Kalberla 2007; Kalberla \& Haud 2006; Lockman et al. 2002; Brüns et al. 2000). The sensitivity goal was set to match or exceed the LAB survey, but with higher angular resolution. The survey completely covers the sky with Nyquist sampling so that the data can be used to provide short-spacings for interferometric imaging.

GASS was designed to meet these criteria by fully sampling Galactic $\mathrm{H}$ I in the velocity range $-400 \leq v \leq 500 \mathrm{~km} \mathrm{~s}^{-1}$ over the sky south of $\delta=1^{\circ}$ with angular resolution of $16^{\prime}$ and a velocity resolution of $1 \mathrm{~km} \mathrm{~s}^{-1}$. The survey used in-band frequency-switching, described below, to maximize observing efficiency while preserving sensitivity to extended emission. Integration times were chosen to achieve a brightness temperature noise $(1 \sigma) \leq 70 \mathrm{mK}$ per channel thus matching the LAB sensitivity for extended sources while having four times the sensitivity of the LAB survey for unresolved sources.

\subsection{Observations}

GASS observations were made with the $21 \mathrm{~cm}$ multibeam receiver system on the Parkes 64 $\mathrm{m}$ radio telescope. The $21 \mathrm{~cm}$ multibeam has 13 dual linear polarization receivers in the single cooled dewar. The 13 feed horns are arranged in a hexagonal pattern at the focal plane, consisting of a central feed, an inner ring of six feeds and an outer ring with an additional six feeds (see Staveley-Smith et al. 1996). At $\lambda=21 \mathrm{~cm}$, the average measured beamwidths for the multibeam are: $14.0^{\prime}$ for the central beam, $14.1^{\prime}$ for beams two to seven with an ellipticity of 0.03 , and $14.5^{\prime}$ for

beams eight to 13 with an ellipticity of 0.06 (Stavelev-Smith et al. 1996). The mean beamwidth is therefore $14.3^{\prime}$ with a separation between adjacent beam centers of $29.1^{\prime}$. If the receiver is oriented at an angle of $19.1^{\circ}$ with respect to the scan direction it produces equally spaced tracks with the inner seven beams.

Observations were conducted between 28 January 2005 and 1 November 2006 in eight observing sessions of typically two weeks in duration. Observing sessions were organised to cover a contiguous region of the sky, usually several hours in right ascension and all declinations. All observations were conducted at night and at elevations greater than $30^{\circ}$, which is the Parkes elevation limit.

GASS consists of two complete surveys of the sky: one scanned in declination, one scanned in right ascension. The receiver angle was adjusted every $5 \mathrm{~s}$ to ensure that the individual beams of the multibeam produce approximately equally spaced tracks on the sky parallel to the scan direction. GASS was designed to be fully sampled with only the inner seven beams to simplify future stray radiation corrections, but data from all 13 beams are used for the results presented here.

Spectra were obtained using a special purpose correlator mode, combining the Multibeam correlator and the Wideband correlator to achieve $8 \mathrm{MHz}$ of bandwidth divided into 2048 channels for both polarizations on all 13 beams. The data were recorded with "in-band" frequency-switching, in which spectra are recorded at two closely spaced frequencies (IFs) centered at 1418.8435 and 
1421.9685 MHz with a $5 \mathrm{~s}$ duty cycle. Frequency-switching allows one to effectively remove the continuum signal and time variations in the system gain. In-band frequency switching, where both bands contain the $\mathrm{H}$ I line, has the added benefit of maximising on-source time because the two spectra can be combined to yield a total about $4.5 \mathrm{MHz}$ or $950 \mathrm{~km} \mathrm{~s}^{-1}$ of continuous bandwidth. The channel spacing is $\Delta v=0.82 \mathrm{~km} \mathrm{~s}^{-1}$ and the effective channel width is $1.0 \mathrm{~km} \mathrm{~s}^{-1}$. Doppler tracking was not applied on-line so the LSRK velocity range accessible varies slightly for each spectrum. The frequency switch of $3.125 \mathrm{MHz}$ corresponds to $660 \mathrm{~km} \mathrm{~s}^{-1}$, so every real emission line feature has an associated, spurious, negative image displaced by $\pm 660 \mathrm{~km} \mathrm{~s}^{-1}$, but most of these negative images fall outside the velocity coverage of the survey. We discuss this further in $\S 3.1 .3$,

A scan consists of all the data obtained while driving the telescope through 8 degrees of right ascension or declination at a rate of $1 \mathrm{deg} \mathrm{min}^{-1}$. It is composed of 26 independent sub-scans from the 13 beams and two polarizations with data samples every $5 \mathrm{~s}$. With these scanning and sampling rates the spacing between adjacent samples is $5^{\prime}$, which compares favourably with the Nyquist sampling, $\lambda / 2 D=5.6^{\prime}$ for an observing wavelength of $\lambda=21 \mathrm{~cm}$ and the dish diameter of $D=64 \mathrm{~m}$. The separation between adjacent beam tracks with a $19.1^{\circ}$ receiver rotation angle is $9.4^{\prime}$. For Nyquist sampling interleaved scans were required. Consecutive scans were offset by $32^{\prime}$ so that adjacent beam tracks were observed with different receivers. An example of the scanning pattern for three interleaved scans is shown in Figure 1, After three scans the spacing between adjacent tracks is $3.1^{\prime}$. On-the-fly observing and subsequent gridding broadens the effective telescope beamwidth to $16^{\prime}$. The integration time per spectrum (pixel) was 30 seconds for the final data product.

\subsection{Data Reduction}

The majority of the data reduction was carried out with Livedata and Gridzilla softwar£ 1 specifically designed to process multibeam data. Livedata performs bandpass correction and flux calibration and Gridzilla produces gridded images from the calibrated, corrected data.

\subsubsection{Bandpass Correction: GASS Mode of Livedata}

The Livedata processing pipeline was developed and used for the HIPASS survey as described by Barnes et al. (2001). In its original form, Livedata derived a bandpass solution from the emissionfree portions of a scan. However, neither this nor similar techniques are suitable for GASS because Galactic H I emission covers the whole sky.

From its origin, Livedata established the use of robust estimation, based mainly on the use of

\footnotetext{
${ }^{1}$ Binaries and source code are available from http://www.atnf.csiro.au/computing/software/livedata.html
} 
median statistics, and this was significant in ameliorating the effects of radio frequency interference (RFI) in the HIPASS survey. For GASS processing, Livedata was adapted to use similar robust techniques for bandpass calibration of frequency-switched Galactic H I data. It works with the quotient of each frequency-switched pair, carefully masking any emission, both spectrally and in the time domain, before determining the average bandpass solution for each scan. The bandpass is calibrated separately for each beam and polarization.

In this section we describe in detail the procedures used to perform the bandpass correction for each beam and polarization, including the formation of quotient spectra, masking emission spectrally and temporally and fitting the average bandpasses. These steps are also graphically outlined in Figure 2. We first define some variables. Let subscripts $i_{1}$ and $i_{2}$ denote the first and second integration in pair number $i$. The spectrum centred at $1418.8435 \mathrm{MHz}$ is $S_{i_{1}}(\nu)$, and the next integration, $S_{i_{2}}(\nu)$, is centered at $1421.9685 \mathrm{MHz}$. The first integration, $S_{i_{1}}(\nu)$, is divided by $S_{i_{2}}(\nu)$ to produce a quotient spectrum $q_{i_{1}}(\nu)$, and vice versa to produce $q_{i_{2}}(\nu)$. In general we use the variable $q$ to denote quotient spectra, $\hat{q}$ to denote time averages of quotient spectra, and $Q$ to denote estimations of the baseline, which may be medians or polynomial fits. Steps in the first and second passes, as described below, are distinguished by the use of prime such as, $q^{\prime}$ and $q^{\prime \prime}$.

Considering the first in each pair of quotients, $q_{i_{1}}(\nu)$, a time-averaged value in emission-free regions for the eight minute scan is determined for each channel. This is ultimately fit to provide the final bandpass solution, $Q^{\prime \prime}(\nu)$. Because $q_{i_{1}}(\nu)$ and $q_{i_{2}}(\nu)$ are reciprocals, the bandpass solution need only be computed for one or the other. Much of the complexity of the algorithm relates to the identification of line emission and RFI and the construction of masks that this identification entails. These masks are determined iteratively in two passes.

The first pass begins with the determination of a base level and characteristic deviation in emission-free regions. The median value over $\nu$ of $q_{i_{1}}(\nu)$ is computed for each $i$ in the scan. The median of these medians then gives the median of quotients, $Q_{1}$, a single number that provides a zeroth-order approximation to the base-level of $q_{i_{1}}(\nu)$ over frequency and time. Normally $Q_{1}$ will be close to unity. Now for each $i$, we compute the median value over $\nu$ of $\left|q_{i_{1}}(\nu)-Q_{1}\right|$. The median of these medians is the median quotient deviation, $D_{1}$. This is used together with $Q_{1}$ to identify line emission or absorption when selecting data to form a time-average quotient value for each channel. Thus, we consider each channel of $q_{i_{1}}(\nu)$ in turn for all $i$ in the scan; i.e. as a function of time. We reject $q_{i_{1}}(\nu)$ for statistical purposes if $\left|q_{i_{1}}(\nu)-Q_{1}\right|>3 D_{1}$. The rejected $q_{i_{1}}(\nu)$ form a time mask for the spectral channel indicating where the scan has passed through a source or encountered transient RFI.

The time mask is then subjected to a broadening algorithm that aims to exclude the low-level wings of the source profile. This step recognizes that masking by means of a discriminant only accounts for the central part of an emission line or RFI. The choice of time masking broadening parameters is a careful balance between defining a mask that is too small so that the diffuse wings of sources are fit as bandpass and the source is clipped or defining a mask that is too large so that 
many masks merge together and the majority of a scan is masked. Many values for the time mask were tested before settling on parameters such that each patch of consecutive false values (rejected samples) in the mask is extended by one on each side if it is at least 2 elements wide, and by a further one on each side for every additional 4 elements. Thus a single isolated false value in the mask is left alone on the basis that it is probably a noise spike. Isolated patches that consist of $1,2,3,4,5,6,7,8,9, \ldots$ consecutive false values will broaden to $1,4,5,6,7,10,11,12,13, \ldots$ samples. As the patch size increases the limiting value of the broadening factor is $150 \%$. In this process it is not uncommon for neighbouring masked regions to blend into a single, larger masked region. An example of the first pass time-masked quotient spectrum is shown in the third panel of Figure 2 ,

After broadening the time mask, or even beforehand, there will usually be some channels for which too few quotients remain to compute a meaningful time-average value; typically this occurs at low H I velocities. If fewer than $90 \%$ of quotients for a given channel are rejected, a first approximation to the bandpass solution for the channel, $\hat{q}_{1}^{\prime}(\nu)$, is computed as the median value of those remaining. If more than $90 \%$ are rejected, then this channel is masked for the entire scan forming a channel mask. Channel masks are then subject to the same broadening process as the time masks, though with more aggressive broadening parameters: the mask is extended by one channel on each side for the first false value, and by a further one on each side for every additional 2 false values. Thus an isolated patch of $1,2,3,4,5,6, \ldots$ false values becomes $3,4,7,8,11,12, \ldots$ with a limiting broadening factor of $200 \%$. Once again, these masking values were chosen as a compromise between channel masks that are so small that real emission in the line wings is included in the fit and masks that are so large that a significant fraction of the band is masked and as a consequence the fit is poorly constrained. Livedata also allows for the channel mask to be augmented manually by specifying up to ten pairs of channel ranges not subject to mask broadening. A single $82 \mathrm{~km} \mathrm{~s}^{-1}$ wide channel mask centered near $0 \mathrm{~km} \mathrm{~s}^{-1}$ was applied to each IF.

Broadening of the channel mask is more exaggerated than for the time mask to avoid removing spectral line wings. As they are of scientific interest, it is also important that the wings not be included in, and therefore potentially removed by, the polynomial baseline fit which is applied in the next step. Channel masking results in gaps in $\hat{q}_{1}^{\prime}(\nu)$ which tend to coincide with channels of particular interest. At low velocities the $\mathrm{H}$ I line occupies the whole scan; baseline information in these channels is effectively lost and can only be estimated by interpolation of neighbouring channels. In practice $\hat{q}_{1}^{\prime}(\nu)$ derived from observed data, and shown in Figure 3, deviates by a few percent from unity. Consequently we found that a robust polynomial fit of degree 15 was required to interpolate across the masked channels and fit the baseline accurately. Robust polynomial fitting for GASS is an iterative process whereby the polynomial is fit to the unmasked channels and points outside $3 \times$ the median absolute deviation from the median are excluded from the second and final iteration. A second approximation, $Q_{1}^{\prime}(\nu)$, to the bandpass solution is thus obtained, thereby completing the first pass.

We found that the high order polynomial fit to $\hat{q}_{1}^{\prime}(\nu)$ works well for most of the sky, but for regions where the line emission is spectrally broad the fit is poorly constrained under the line. This 
is mainly an issue towards the Galactic plane and results in errors at the 3-5\% level as discussed in 3.1.2. Lower order polynomials were also tested but we found that they were not able to fit the substructure of the quotient spectrum.

The quotients and fits for an example scan in the direction of the Magellanic Stream are shown in Figure 3. This scan is a particularly difficult case because the Galactic and Magellanic Stream emission are spectrally near to each other with very little spectral baseline between. The top panel of Figure 3 shows the results of the first pass, where the masked, time-averaged quotient spectrum, $\hat{q}_{1}^{\prime}(\nu)$, is plotted together with its polynomial fit, $Q_{1}^{\prime}(\nu)$. The plotted quotient spectrum demonstrates clearly the need for the 15 th degree polynomial fit. The polynomial fit in this panel is, however, not perfect, caused to a large extent by the parts of the spectral line that are not yet masked.

The lower panel in Figure 3 shows the results of a second pass through the data, which improves the masking of emission. The second pass essentially repeats the first pass except that $Q_{1}$ is replaced with $Q_{1}^{\prime}(\nu)$, which provides a channel-specific value. $D_{1}$ is recomputed accordingly but the discriminant for time masking is set at $2 D_{1}$ (rather than $3 D_{1}$ ) because $Q_{1}^{\prime}(\nu)$ is a more reliable estimate of the base-level than $Q_{1}$. The new time average of the more effectively masked quotient spectra, $q_{1}^{\prime \prime}(\nu)$, is shown in the lower panel of Figure 3. This spectrum is once again fit with a robust polynomial of degree 15 to give the final bandpass solution, $Q_{1}^{\prime \prime}(\nu)$, as shown in Figure 3 , Here we can see that the more extensive masking results in a better fit to the off-line portions of the spectrum, although deviations remain at the $\sim 0.1 \%$ level.

The bandpass corrected spectra are thus calculated from $Q_{1}^{\prime \prime}(\nu)$ as:

$$
\begin{aligned}
& S_{i_{1}}^{\prime}(\nu)=\operatorname{norm}\left(q_{i_{1}}(\nu) / Q_{1}^{\prime \prime}(\nu)\right) T_{i_{1}}-\bar{T}_{1}, \\
& S_{i_{2}}^{\prime}(\nu)=\operatorname{norm}\left(q_{i_{2}}(\nu) \times Q_{1}^{\prime \prime}(\nu)\right) T_{i_{2}}-\bar{T}_{2},
\end{aligned}
$$

where norm() indicates normalization to unit value, $T_{i_{1}}$ and $T_{i_{2}}$ are the system temperatures for the two spectra, and $\bar{T}_{1}$ and $\bar{T}_{2}$ are the median values of $T_{s y s}$ for the two frequency-switched pairs evaluated over all $i$ in the scan.

\subsubsection{RFI Flagging}

Occasional, weak, narrow-line RFI appears at a fixed topocentric frequency in much or all of some scans. Once Doppler-shifted to the LSRK, such RFI produces low-level features that appear to move across the sky in successive velocity channels. Because of frequency-switching, such features may be negative as well as positive.

Narrow-line RFI appears in only one or two channels, but may cause ringing in adjacent channels. The first step in flagging RFI is to compute, for each channel, the time-average value of $S_{i_{1}}^{\prime}(\nu)$ for all $i$ in the scan. From this, the running mean computed over 21 channels honouring the channel mask determined previously, is subtracted. The result is converted to a mask with values 
$-1,0$, or +1 , depending on whether the channel value is outside $6 \times$ the median absolute deviation; the magnitude of the departure from zero is not considered, only the sign.

The mask is then scanned for non-zero channels that should be preserved, the remainder being flagged as likely RFI. Consecutive non-zero channels of the same sign are preserved if there are more than 2 of them in the sequence, the width test, or if they are close to such a sequence of the same sign. Here, "close" means that there is no intervening value of the opposite sign, and there is no intervening sequence of consecutive zeroes of length greater than 5 channels. This closeness test is intended to protect outliers in the wings of real emission lines. This algorithm proved effective in removing the majority of the RFI that appeared in spectral datacubes.

\subsubsection{Post-processing}

Low-level baseline residuals may remain in each spectrum at this point because the original bandpass solution was computed as a time-average over the whole scan. This residue was removed separately for each spectrum by subtracting a 10-th order polynomial fit from the spectrum for high Galactic latitude areas $\left(b>10^{\circ}\right)$ and a simple median level fit from areas within 10 degrees of the Galactic plane. The algorithm was as described previously, using a mask derived separately for each spectrum, including the user-defined mask as before. We discuss baseline quality in $\S 3.1$.

Finally, the spectra were Doppler-shifted to transform them to the LSRK velocity frame for gridding and analysis. The scheme used for Doppler correction was to rescale the reference frequency and channel spacing by the Doppler factor, and then Fourier-shift the spectrum, usually by less than one channel, so that the reference frequency was an integer factor of the original channel spacing $(3.90625 \mathrm{kHz})$. This scheme is employed by Gridzilla (see below) and allows the combination of spectra taken months or years apart without requiring interpolation of the frequency axis.

\subsubsection{Brightness Calibration}

First order brightness calibration was applied on-line through injection of noise from a diode switched with a frequency of $500 \mathrm{~Hz}$. Average on-line system temperatures for each beam and polarization were recorded along with each $5 \mathrm{~s}$ spectrum. Typical system temperature values are between $21-23 \mathrm{~K}$.

Before imaging, spectra are converted to beam averaged brightness temperature, $T_{B}$, from observations of the IAU standard line calibration regions S6, S8, and S9 (Williams 1973; Kalberla et al. 1982). One of the three standard line regions was observed each day. Observations were conducted by placing each of the thirteen beams on the region in turn. The peak of the observed line was used to calculate calibration scaling factors for each beam and each polarization assuming peak brightness temperatures of $T_{B}=83 \mathrm{~K}$ for $\mathrm{S} 9, T_{B}=53 \mathrm{~K}$ for $\mathrm{S} 6$ and $T_{B}=76 \mathrm{~K}$ for $\mathrm{S} 8$ valid for the 
Parkes beam (Kalberla et al. 1982; Brüns et al. 2005). One set of calibration factors was calculated for each observing session and showed rms variations between observing sessions of $1-2 \%$ on most beams. The notable exceptions are the second polarization on beams 10 and 12, which were known to have unstable low noise amplifiers. The calibration factors for these beams varied by as much as $6 \%$ over the 21 months of the survey. The overall effect on the data was found to be minimal and these beams were included in the final data cubes. These brightness temperature calibration factors were applied within Livedata following bandpass calibration.

To check that our calibration factors were not affected by the observing strategy of pointed calibrator observations rather than the on-the-fly scans that were used for the full survey, we observed S9 in on-the-fly mode as well. Calibration factors determined from these scans were fully consistent with the factors determined from pointed observations.

\subsubsection{Imaging}

Imaging was performed by Gridzilla, a statistical gridder developed for the HIPASS survey and subsequently extended for more general use with Parkes multibeam and other single-dish data. The algorithm is described by Barnes et al. (2001). Part of Gridzilla's later development involved adding full support for FITS celestial and spectral world coordinate systems (Greisen \& Calabretta 2002; Calabretta \& Greisen 2002; Greisen et al. 2006). In particular, we used the Zenithal Equal-Area projection (ZEA) as the most appropriate choice for mapping the hemisphere.

For each pixel in the output data cube, Gridzilla computes a weight for each input spectrum based on its angular distance from the pixel. For GASS, it then calculated the pixel value from the spectral values and weights using weighted median estimation, which is robust against RFI and other artifacts. The weighted median of a set of measurements is the middle-weight value - the sum-of-weights of all measurements less than it being equal to that of all measurements greater; pro rata interpolation being used to bisect the sum-of-weights if required.

For the weighting function we used natural beam weighting, with the beam modelled by a 2D Gaussian of FWHM 14'.4, combined with an additional 2D Gaussian of FWHM 14', with a cutoff radius of $8^{\prime}$. This combination of weighting functions degrades the angular resolution of the images slightly below the telescope FWHM of $14^{\prime} .4$ but produces smoother and more sensitive images. Measurements of unresolved sources inserted into the data prior to gridding show that the resulting resolution is $\sim 16^{\prime}$.

\section{Data Products}

The primary data product for this first release is a data cube $(\alpha, \delta, v)$ of the entire survey region and full velocity range without stray radiation correction. The data were gridded in a ZEA 
projection covering $\delta<1^{\circ}$ with the South Celestial Pole at the center of the image. The velocity range covered by the gridded cube is $-400 \mathrm{~km} \mathrm{~s}^{-1} \leq V_{L S R} \leq 500 \mathrm{~km} \mathrm{~s}^{-1}$.

A comprehensive view of GASS is given in Figure 4, which is a combination of moment maps created in $\sim 40 \mathrm{~km} \mathrm{~s}^{-1}$ intervals over the full velocity range, color-coded by velocity. Most of the key features of the Southern sky are clearly visible in this image including the Galactic plane, Magellanic Clouds, Magellanic Stream and Leading Arm, several high velocity cloud complexes, and a few galaxies belonging to the Sculptor group.

An image of the total column density, with an Aitoff projection in Galactic coordinates, is shown in Figure 5. The image has been calculated from the zeroth moment over the velocity range $-400 \leq V_{L S R} \leq 500 \mathrm{~km} \mathrm{~s}^{-1}$ and converted to units of $\times 10^{21} \mathrm{~cm}^{-2}$ using the usual optically thin scaling factor $1.8 \times 10^{18} \mathrm{~cm}^{-2} \mathrm{~K}^{-1} \mathrm{~km}^{-1} \mathrm{~s}$. This image is dominated by emission at local velocities.

We show three individual velocity channels of low velocity $\left(\left|V_{L S R}\right| \lesssim 30 \mathrm{~km} \mathrm{~s}^{-1}\right)$ gas in Figures [6] - 8 that highlight the small-scale structure visible in the GASS data. Similarly, Figures 9 and 10 are images of the high and intermediate velocity sky, where high velocity gas is defined as $\left|V_{L S R}\right| \geq 100 \mathrm{~km} \mathrm{~s}^{-1}$ and intermediate velocity gas is defined as $40 \leq\left|V_{L S R}\right|<100 \mathrm{~km} \mathrm{~s}^{-1}$. The negative high velocities are dominated by Galactic emission near to the Galactic center and a portion of the Magellanic Stream, whereas the positive velocities are dominated by Galactic plane emission and the Large and Small Magellanic Clouds. The intermediate velocity sky is dominated by Galactic emission, however there are some interesting filamentary extensions off the Galactic plane. Small HVCs and IVCs are visible in both figures.

A summary of the survey specifications is given in Table1. Spectra from the survey and information about other data products available for download can be found at http://www.atnf.csiro.au/research/GASS.

\subsection{Data Quality}

The typical rms per channel in the cube is $\sim 57 \mathrm{mK}$. This is determined from an image of the rms noise across the full survey region, as calculated from the median of the noise in nine blocks of $\sim 20$ line-free channels. The rms image is shown in Figure 11, This approach effectively removes contamination of the rms measurement from negative residuals (see $\S$ 3.1.3) from the Magellanic system and Galactic Center region in the otherwise line-free channels. The noise is relatively smooth across the majority of the sky, with a mode of $57 \mathrm{mK}$. As seen in Fig. 11, the rms around the Galactic plane region is clearly higher than the rest of the sky. This is due to both strong continuum sources and the large fraction of the band filled by $\mathrm{H}$ I emission in the Galactic plane. Both effects raise the system temperature. Similar increases in rms occur in the region of the LMC. The rms is lower in the overlap region between scans and also where scans were repeated, such as the large area near $1 \mathrm{~h}$ of right ascension. Assuming a rms brightness temperature of $57 \mathrm{mK}$, the $1 \sigma$ column density noise in a single velocity channel is $1 \times 10^{17} \mathrm{~cm}^{-2}$. For a typical HVC of width 
$30 \mathrm{~km} \mathrm{~s}^{-1}$, the $3 \sigma$ sensitivity limit is $N_{H I}=1.6 \times 10^{18} \mathrm{~cm}^{-2}$.

There are three extended areas where portions of the spectra in the range velocities $\sim 230-320$ $\mathrm{km} \mathrm{s}^{-1}$ have increased noise by a factor of $\sqrt{2}$. These regions result from two days' worth of RA scans that were flagged because they exhibited nearly continuous, broadband RFI of unknown origin. The flagged scans lie in the regions: $11^{\mathrm{h}} 15^{\mathrm{m}} \lesssim \alpha \lesssim 12^{\mathrm{h}} 15^{\mathrm{m}},-25^{\circ} \lesssim \delta \lesssim 0^{\circ} ; 11^{\mathrm{h}} \lesssim \alpha \lesssim 13^{\mathrm{h}}$, $-65^{\circ} \lesssim \delta \lesssim-45^{\circ}$; and $13^{\mathrm{h}} 45^{\mathrm{m}} \lesssim \alpha \lesssim 15^{\mathrm{h}} 15^{\mathrm{m}},-78^{\circ} \lesssim \delta \lesssim-70^{\circ}$. These areas are marginally visible in Figure 11 as regions of increased noise.

\subsubsection{Stray Radiation}

A fundamental limitation of GASS data in the present release is stray radiation: $\mathrm{H}$ I emission that enters the receiver from the sidelobes of the telescope rather than through the main beam. Kalberla et al. (1980) has shown that stray radiation can make a significant contribution (15-50\% of the profile area) to observed Galactic $\mathrm{H}$ I emission profiles, mostly at high Galactic latitudes where emission in the primary beam is weak. The LAB survey has been corrected for stray radiation, and the second GASS data release will be corrected as well, but at this stage we have simply estimated the total amount stray radiation in GASS for various regions of the sky. This was done by convolving the GASS and LAB surveys to $2^{\circ}$ angular resolution, computing the total column density over their common velocity range $\left(-400 \mathrm{~km} \mathrm{~s}^{-1} \leq V_{L S R} \leq 400 \mathrm{~km} \mathrm{~s}^{-1}\right)$, and interpreting the difference as stray radiation in GASS. Figure 12 shows the convolved GASS column density, the estimated stray column, and the stray fraction. For most lines of sight the fraction of the total column density believed to arise from stray radiation is between 5 and $15 \%$, with some particularly low column density regions showing fractions as high as $35 \%$.

To demonstrate the spectral behaviour of the stray radiation component we compared LAB and GASS spectra towards several regions representative of high stray radiation fraction, low stray fraction, and average stray fraction, as calculated above. The regions are marked on Figure 13 and the spectra are shown in Figure 14. These spectra are averaged over $5 \times 5$ degrees in both surveys to minimize any resolution-dependent effects. For an area with average stray fraction it is clear that the GASS spectrum reproduces the stray radiation corrected LAB spectrum very well, with small departures in the line wings where the total intensity is small. The high stray fraction spectrum was extracted towards a region where the fraction of the total column density attributed to stray radiation was on the order of $30 \%$. In this area the GASS spectrum has a very low peak brightness temperature of $\sim 1.9 \mathrm{~K}$, and yet the LAB spectrum peak is even lower at $\sim 1.4 \mathrm{~K}$. The GASS spectrum also shows significant effects of stray radiation in the line wings. 


\subsubsection{Baseline Quality}

Although GASS spectral baselines are generally very good, there are residual ripples in the final baselines with maximum peak-to-peak variations of $\sim 50 \mathrm{mK}$. It was the presence of much larger $(\sim 100-200 \mathrm{mK})$ bandpass amplitude ripples and striping in test images that necessitated the use of high-order polynomials in the bandpass correction algorithm as described in 92 , These were effectively removed by the post-bandpass polynomial fits applied to scans away from the Galactic Plane. To ensure that the high-order polynomials had not subtracted real emission we compared spectra obtained from GASS to spectra obtained from the LAB survey. These showed generally very good agreement at the $\sim 50-70 \mathrm{mK}$ level. Several examples are shown in Figure 15, demonstrating the baseline quality of GASS.

Baseline quality deteriorates in some regions toward the Galactic Plane and the Magellanic Clouds. The bandpass correction method described in 2.3 .1 is limited in cases where the $\mathrm{H}$ I emission in the quotient spectrum fills $\sim 50 \%$ of the band. In these cases the polynomial fit to the quotient spectrum is poorly constrained and therefore the bandpass under the line is not well determined. This results in some small, $\sim 3-5 \%$, baseline errors where the measured line temperatures can appear either too large or too small relative to spectra from LAB. Examples are shown in Figure 16, where the GASS spectra show higher and lower peak values than LAB.

\subsubsection{Other Artifacts}

As discussed in $\S 2.2$, the in-band frequency-switching will produce a negative copy of a spectral line displaced by $\pm 660 \mathrm{~km} \mathrm{~s}^{-1}$. As a practical matter, most of these artifacts lie outside the final data cube, but there are some notable exceptions for features at $V_{L S R}>260 \mathrm{~km} \mathrm{~s}^{-1}$ (with the inverted feature appearing at negative velocities) and $V_{L S R}<-160 \mathrm{~km} \mathrm{~s}^{-1}$ (at positive velocities). This is particularly evident towards the Galactic Center, the Magellanic Clouds (apparent at extreme negative velocities) and the Northern tip of the Magellanic Stream (apparent at extreme positive velocities) as can be seen in the bottom panel of Figure 16.

Another artifact appears as a low amplitude grid-like scanning pattern in channel images with low-level emission. This is due to residual ripples in the bandpass of individual scans, which can cause slight offsets between adjacent scans. These are typically within the noise, but because they are spatially correlated they can be seen in the rms image in Figure 11.

\section{Summary}

The Parkes Galactic All-Sky Survey (GASS) is a high spectral and angular resolution H I line survey of the sky south of $\delta=1^{\circ}$. The first-stage data release includes a full cube at $16^{\prime}$ angular resolution, $1.0 \mathrm{~km} \mathrm{~s}^{-1}$ spectral resolution and $\sim 57 \mathrm{mK} \mathrm{rms}$ noise over the velocity range 
$-400 \mathrm{~km} \mathrm{~s}^{-1} \leq V_{L S R} \leq 500 \mathrm{~km} \mathrm{~s}^{-1}$. Spectra from this cube and several other data products are now publicly available at http://www.atnf.csiro.au/research/GASS. Table 2 gives a comparison of GASS with other large-scale surveys of Galactic H I. At southern declinations GASS is unsurpassed in sensitivity and, outside of the Galactic plane, in angular and velocity resolution. The improvement of GASS over the LAB survey is illustrated by the spectra in Figure 15, shown at the full angular resolution of both surveys.

The current release of GASS is intended to provide data that are ideal for study of high velocity $\left(\left|V_{L S R}\right| \gtrsim 100 \mathrm{~km} \mathrm{~s}^{-1}\right) \mathrm{H} \mathrm{I}$, where the effects of stray radiation are negligible, and smallscale features, where the angular resolution is a significant advance. A complete catalog of HVCs and IVCs from GASS is in preparation by D. J. Pisano et al. (2009, in preparation). Because the current data release has not been corrected for stray radiation, care should be taken when using it to derive global Milky Way properties, especially at low column densities where there can be significant amounts of stray radiation. For users interested in total column densities measured to extragalactic sightlines these measurements should be considered in conjunction with the total fraction of the column due to stray radiation (Figure 12).

GASS data have already been used for several of the scientific areas described in \$1 Using data from the first year of observations McClure-Griffiths et al. (2006) found that one of the largest Galactic supershells, GSH 242-03+37, is in fact a chimney, with evidence for breakout on both sides of the Galactic plane. GSH 242-03+37 appears capped by thin, clumpy filaments of $\mathrm{H}$ I emission at heights of $z \sim 1.5 \mathrm{kpc}$ above the Galactic midplane. Those authors suggested that the clumpy filaments may be the precursor of halo clouds detected by Lockman (2002).

Ford et al. (2008) used GASS data to extend our knowledge of Galactic halo clouds by constructing a catalogue of hundreds of clouds in a $720 \mathrm{deg}^{2}$ GASS pilot region centred on $l=335^{\circ}$, $b=0^{\circ}$. Though restricted to a small range of Galactic longitudes, they found that the distribution of clouds is significantly peaked at a Galactocentric radius of $3.75 \mathrm{kpc}$ and that the clouds are associated with loops and filaments consistent with a chimney origin as suggested for GSH 242-03+37. The forthcoming complete catalog by H. A. Ford et al. (2009, in preparation) will further explore the Galactic distribution of these clouds.

Finally, McClure-Griffiths et al. (2008) have used GASS data to study the interaction of an HVC in the Magellanic Leading Arm with the Galactic disk. They showed that the Leading Arm crosses the Milky Way disk at a Galactocentric radius of $17 \mathrm{kpc}$, which is close to the interaction region predicted in older models (e.g., Connors et al. 2006; Yoshizawa \& Noguchi 2003) of the Magellanic System but somewhat surprising given the revised proper motions for the Large and Small Magellanic Clouds (van der Marel et al. 2002; Kallivavalil et al. 2006).

As a new, sensitive survey of Galactic H I, GASS is already producing excellent results and will be a valuable database. Work is underway to produce a stray-radiation corrected version of GASS for a second data release. 
We acknowledge the great dedication of the ATNF staff at Parkes and Marsfield towards making the special observing mode for this project available and supporting our observations. We especially thank Malte Marquarding for assisting with various software challenges during the data analysis. Thanks also to Bill Saxton for his assistance in creating Figure 2. D.J.P. acknowledges partial support for this project from NSF grant AST0104439 and thanks the ATNF for its generosity and hospitality through the Distinguished Visitor program. S.J. thanks the NSF for support through the Research Experiences for Undergraduates program at NRAO. T.M. acknowledges the support of an ARC Australian Postdoctoral Fellowship (DP0665973). P.K. and L.D. acknowledge support from Deutsche Forschungsgemeinschaft, grant KA1265/5-1. The Parkes Radio Telescope is part of the Australia Telescope which is funded by the Commonwealth of Australia for operation as a National Facility managed by CSIRO.

Facilities: Parkes ()

\section{REFERENCES}

Barnes, D. G. et al. 2001, MNRAS, 322, 486

Blitz, L., Spergel, D. N., Teuben, P. J., Hartmann, D., \& Burton, W. B. 1999, ApJ, 514, 818

Braun, R. \& Burton, W. B. 1999, A\&A, 341, 437

Bregman, J. N. 1980, ApJ, 236, 577

BrüZs, C., Kerp, J., Kalberla, P. M. W., \& Mebold, U. 2000, A\&A, 357, 120

Brüns, C., Kerp, J., Staveley-Smith, L., Mebold, U., Putman, M. E., Haynes, R. F., Kalberla, P. M. W., Muller, E., \& Filipovic, M. D. 2005, A\&A, 432, 45

Calabretta, M. R. \& Greisen, E. W. 2002, A\&A, 395, 1077

Connors, T. W., Kawata, D., \& Gibson, B. K. 2006, MNRAS, 371, 108

de Heij, V., Braun, R., \& Burton, W. B. 2002, A\&A, 392, 417

Delhaye, J. 1965, Galactic structure. Edited by Adriaan Blaauw and Maarten Schmidt. Published by the University of Chicago Press, 61

Ewen, H. I. \& Purcell, E. M. 1951, Nature, 168, 356

Ford, H. A., McClure-Griffiths, N. M., Lockman, F. J., Bailin, J., Calabretta, M. R., Kalberla, P. M. W., Murphy, T., \& Pisano, D. J. 2008, ApJ, in press, (astro-ph/0807.3550)

Gibson, S. J., Taylor, A. R., Higgs, L. A., \& Dewdney, P. E. 2000, ApJ, 540, 851

Gordon, M. A. 1976, Methods of Experimental Physics, 12, 277 
Greisen, E. W. \& Calabretta, M. R. 2002, A\&A, 395, 1061

Greisen, E. W., Calabretta, M. R., Valdes, F. G., \& Allen, S. L. 2006, A\&A, 446, 747

Haud, U. \& Kalberla, P. M. W. 2007, A\&A, 466, 555

Heiles, C. 1984, ApJS, 55, 585

Henderson, A. P., Jackson, P. D., \& Kerr, F. J. 1982, ApJ, 263, 116

Kalberla, P. M. W., Burton, W. B., Hartmann, D., Arnal, E. M., Bajaja, E., Morras, R., \& Poppel, W. G. L. 2005, A\&A, 440, 775

Kalberla, P. M. W. \& Haud, U. 2006, A\&A, 455, 481

Kalberla, P. M. W., Mebold, U., \& Reich, W. 1980, A\&A, 82, 275

Kalberla, P. M. W., Mebold, U., \& Reif, K. 1982, A\&A, 106, 190

Kallivayalil, N., van der Marel, R. P., \& Alcock, C. 2006, ApJ, 652, 1213

Kavars, D. W., Dickey, J. M., McClure-Griffiths, N. M., Gaensler, B. M., \& Green, A. J. 2005, ApJ, 626, 887

Kerr, F. J. 1962, MNRAS, 123, 327

Kuntz, K. D. \& Danly, L. 1996, ApJ, 457, 703

Levine, E. S., Blitz, L., \& Heiles, C. 2006, ApJ, 643, 881

Lockman, F. J. 2002, ApJ, 580, L47

Lockman, F. J. 2003, ApJ, 591, L33

Lockman, F. J., Benjamin, R. A., Heroux, A. J., \& Langston, G. I. 2008, ApJ, 679, L21

Lockman, F. J., Murphy, E. M., Petty-Powell, S., \& Urick, V. J. 2002, ApJ, 140, 331

Maller, A. H., \& Bullock, J. S. 2004, MNRAS, 355, 694

McClure-Griffiths, N. M. \& Dickey, J. M. 2007, ApJ, 671, 427

McClure-Griffiths, N. M., Dickey, J. M., Gaensler, B. M., \& Green, A. J. 2002, ApJ, 578, 176

McClure-Griffiths, N. M., Dickey, J. M., Gaensler, B. M., Green, A. J., Haverkorn, M., \& Strasser, S. 2005, ApJS, 158, 178

McClure-Griffiths, N. M., Ford, A., Pisano, D. J., Gibson, B. K., Staveley-Smith, L., Calabretta, M. R., Dedes, L., \& Kalberla, P. M. W. 2006, ApJ, 638, 196 
McClure-Griffiths, N. M., Staveley-Smith, L., Lockman, F. J., Calabretta, M. R., Ford, H. A., Kalberla, P. M. W., Murphy, T., Nakanishi, H., \& Pisano, D. J. 2008, ApJ, 673, L143

Muller, C. A., Oort, J., \& Raimond, E. 1963, Comptes Rendus l'Academie des Sciences, 257, 1661

Peek, J. E. G., Putman, M. E., McKee, C. F., Heiles, C., \& Stanimirović, S. 2007, ApJ, 656, 907

Peek, J. E. G., \& Heiles, C. 2008, arXiv:0810:1283

Putman, M. E., Bland-Hawthorn, J., Veilleux, S., Gibson, B. K., Freeman, K. C., \& Maloney, P. R. 2003, ApJ, 597, 948

Putman, M. E., de Heij, V., Staveley-Smith, L., Braun, R., Freeman, K. C., Gibson, B. K., Burton, W. B., \& Barnes, D. G. e. a. 2002, AJ, 123, 873

Rector, T., Levay, Z., Frattare, L., English, J., \& Pu'uohau-Pummill, K. 2007, AJ, 133, 598

Smith, G. P. 1963, Bull. Astron. Inst. Netherlands, 17, 203 2006ApJ...644L...1S

Sommer-Larsen, J. 2006, ApJ, 644, L1

Stanimirović, S., Putman, M., Heiles, C., Peek, J. E. G., Goldsmith, P. F., Koo, B.-C., Krčo, M., Lee, J.-J., Mock, J., Muller, E., Pandian, J. D., Parsons, A., Tang, Y., \& Werthimer, D. 2006, ApJ, 653, 1210

Staveley-Smith, L., Wilson, W. E., Bird, T. S., Disney, M. J., Ekers, R. D., Freeman, K. C., Haynes, R. F., Sinclair, M. W., Vaile, R. A., Webster, R. L., \& Wright, A. E. 1996, Publications of the Astronomical Society of Australia, 13, 243

Stil, J. M., Taylor, A. R., Dickey, J. M., Kavars, D. W., Martin, P. G., Rothwell, T. A., Boothroyd, A. I., Lockman, F. J., \& McClure-Griffiths, N. M. 2006, AJ, 132, 1158

Taylor, A. R., Gibson, S. J., Peracaula, M., Martin, P. G., Landecker, T. L., Brunt, C. M., Dewdney, P. E., Dougherty, S. M., Gray, A. D., Higgs, L. A., Kerton, C. R., Knee, L. B. G., Kothes, R., Purton, C. R., Uyaniker, B., Wallace, B. J., Willis, A. G., \& Durand, D. 2003, AJ, 125, 3145

Tripp, T. M., Wakker, B. P., Jenkins, E. B., Bowers, C. W., Danks, A. C., Green, R. F., Heap, S. R., Joseph, C. L., Kaiser, M. E., Linsky, J. L., \& Woodgate, B. E. 2003, AJ, 125, 3122

van der Marel, R. P., Alves, D. R., Hardy, E., \& Suntzeff, N. B. 2002, AJ, 124, 2639

Wakker, B. P., Howk, J. C., Savage, B. D., van Woerden, H., Tufte, S. L., Schwarz, U. J., Benjamin, R., Reynolds, R. J., Peletier, R. F., \& Kalberla, P. M. W. 1999, Nature, 402, 388

Wakker, B. P. \& van Woerden, H. 1997, ARA\&A, 35, 217

Westmeier, T., Brüns, C., \& Kerp, J. 2005, A\&A, 432, 937 
Williams, D. R. W. 1973, A\&AS, 8, 505

Yoshizawa, A. M. \& Noguchi, M. 2003, MNRAS, 339, 1135 
Table 1. Survey parameters

\begin{tabular}{lc}
\hline \hline \multicolumn{1}{c}{ Parameter } & Value \\
\hline Sky coverage & $\delta<1^{\circ}$ \\
Integration time & $30 \mathrm{~s}$ per spectrum \\
Central beam FWHM & $14.0^{\prime}$ \\
Gridded Angular resolution & $16^{\prime}$ \\
Velocity range & $-400<V_{L S R}<500 \mathrm{~km} \mathrm{~s}^{-1}$ \\
Channel Spacing & $0.82 \mathrm{~km} \mathrm{~s}^{-1}$ \\
Channel Width & $1.0 \mathrm{~km} \mathrm{~s}^{-1}$ \\
$1 \sigma T_{B}$ noise & $57 \mathrm{mK}^{18}$ \\
$3 \sigma \mathrm{N}_{H I}$ sensitivity limit & $1.6 \times 10^{\mathrm{a}} \mathrm{cm}^{-2}$ \\
\hline
\end{tabular}

${ }^{\mathrm{a}}$ For $\Delta v=30 \mathrm{~km} \mathrm{~s}^{-1}$.

Table 2. Survey Comparison

\begin{tabular}{|c|c|c|c|c|c|c|}
\hline Survey & Sky Coverage & $\begin{array}{l}\text { Velocity Range } \\
\qquad\left(\mathrm{km} \mathrm{s}^{-1}\right)\end{array}$ & $\begin{array}{c}\text { Beamwidth } \\
\left({ }^{\prime}\right)\end{array}$ & $\begin{array}{c}\Delta \mathrm{v} \\
\left(\mathrm{km} \mathrm{s}^{-1}\right)\end{array}$ & $\begin{array}{c}1 \sigma \mathrm{T}_{B} \\
(\mathrm{mK})\end{array}$ & Reference \\
\hline HIPASS-HVC & $\delta<2^{\circ}$ & $-500<V_{L S R}<500$ & 15.5 & 26.4 & 8 & 1 \\
\hline LAB & Entire sky & $-450<V_{L S R}<400$ & 36 & 1.3 & 70 & 2 \\
\hline \multirow[t]{2}{*}{ SGPS } & $253^{\circ} \leq l \leq 358^{\circ}$, & & & & & \\
\hline & $\begin{array}{c}5^{\circ} \leq l \leq 20^{\circ} \\
|b| \leq 1.5^{\circ}\end{array}$ & $-100<V_{L S R}<126^{\mathrm{a}}$ & 2 & 0.8 & 1600 & 3 \\
\hline GALFA-HI & $-1^{\circ}<\delta<38^{\circ}$ & $-700<V_{L S R}<700$ & 3.5 & 0.18 & $\leq 100$ & 4 \\
\hline GASS & $\delta<1^{\circ}$ & $-400<V_{L S R}<500$ & 16 & 0.82 & 57 & 5 \\
\hline
\end{tabular}

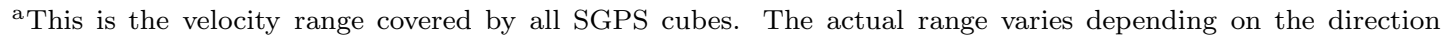
observed and has a maximal coverage of $-300<V_{L S R}<266 \mathrm{~km} \mathrm{~s}^{-1}$.

1 Putman et al. 2002)

2 Kalberla et al. (2005)

3 McClure-Griffiths et al. (2005)

4 Peek \& Heiles 2008)

${ }^{5}$ This Paper 


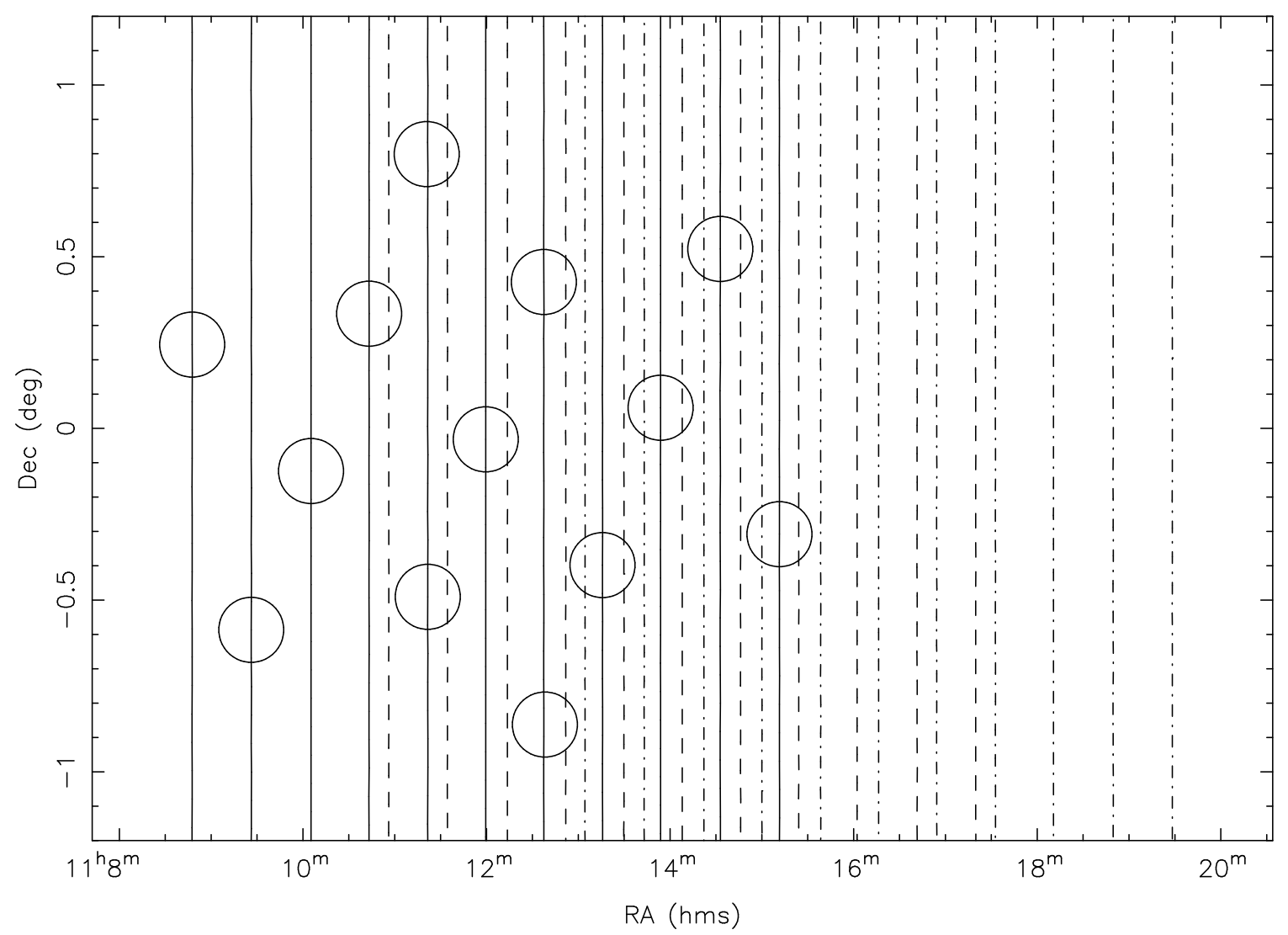

Fig. 1. - Scan pattern for three consecutive scans of the multibeam receiver. The three scans are shown by the solid line, the dashed line and the dash-dotted line. Overlaid on the first scan are circles showing the multibeam hexagonal pattern. The receiver is rotated at $19.1^{\circ}$ with respect to the scan direction, which is the optimal angle for a seven beam system. As a result several outer beams track the same part of sky as the inner beams. 


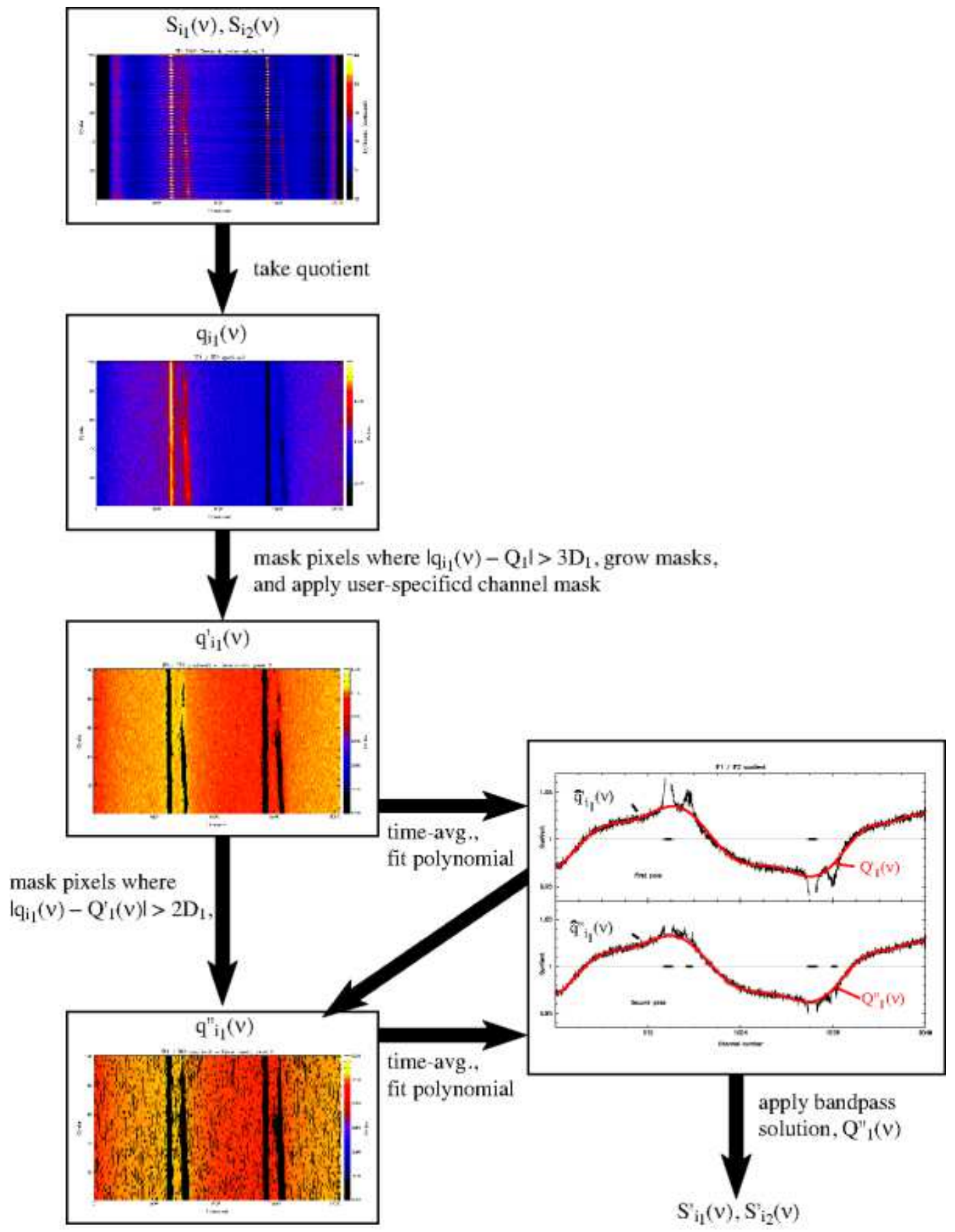

Fig. 2. - Diagram outlining the bandpass solution steps outlined in 2.3.1. Each color panel represents data from a single scan, with time along the ordinate and channel number along the abscissa. The line plots show time-averaged spectra at two stages in the processing. The black and grey solid lines at a quotient value of 1.0 represent the channel masks; the former as derived from the time masks, the latter is the user-defined channel mask. Because the quotients for the two IFs are reciprocals of each other, we have only shown the quotients from the first IF. The resulting solution can be applied to both IFs. 


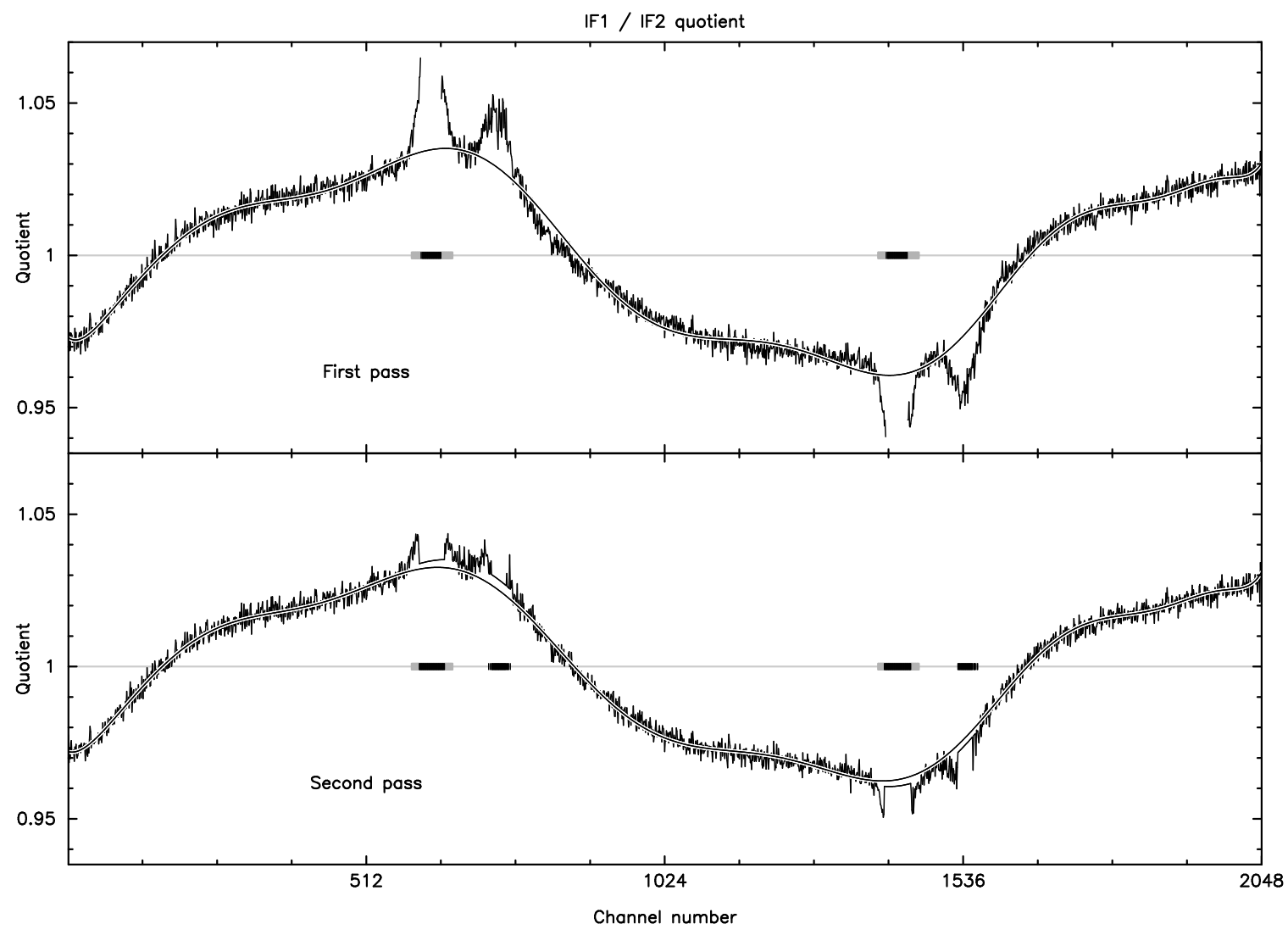

Fig. 3.- Average quotient spectra and their polynomial fits for the two passes of the bandpass solution described in $\$ 2.3 .1$. The top panel shows the time-averaged and masked quotient spectrum, $\hat{q}_{1}^{\prime}(\nu)$, overlaid with its polynomial fit, $Q_{1}^{\prime}(\nu)$. The user-defined channel mask is indicated by the grey solid lines at a quotient value of 1.0, while the black solid lines show the derived channel mask. The polynomial fit in this panel is clearly not perfect, caused to a certain extent by the parts of the spectral line that are not yet masked. The lower panel shows the second pass in which $Q_{1}^{\prime}(\nu)$ is used to refine the time masks and produce a new average, $\hat{q}_{1}^{\prime \prime}(\nu)$. The second pass fit, $Q_{1}^{\prime \prime}(\nu)$, is the bandpass solution. In this panel the polynomial from the first pass is shown in the masked channels to indicate how the second-pass fit differs. 


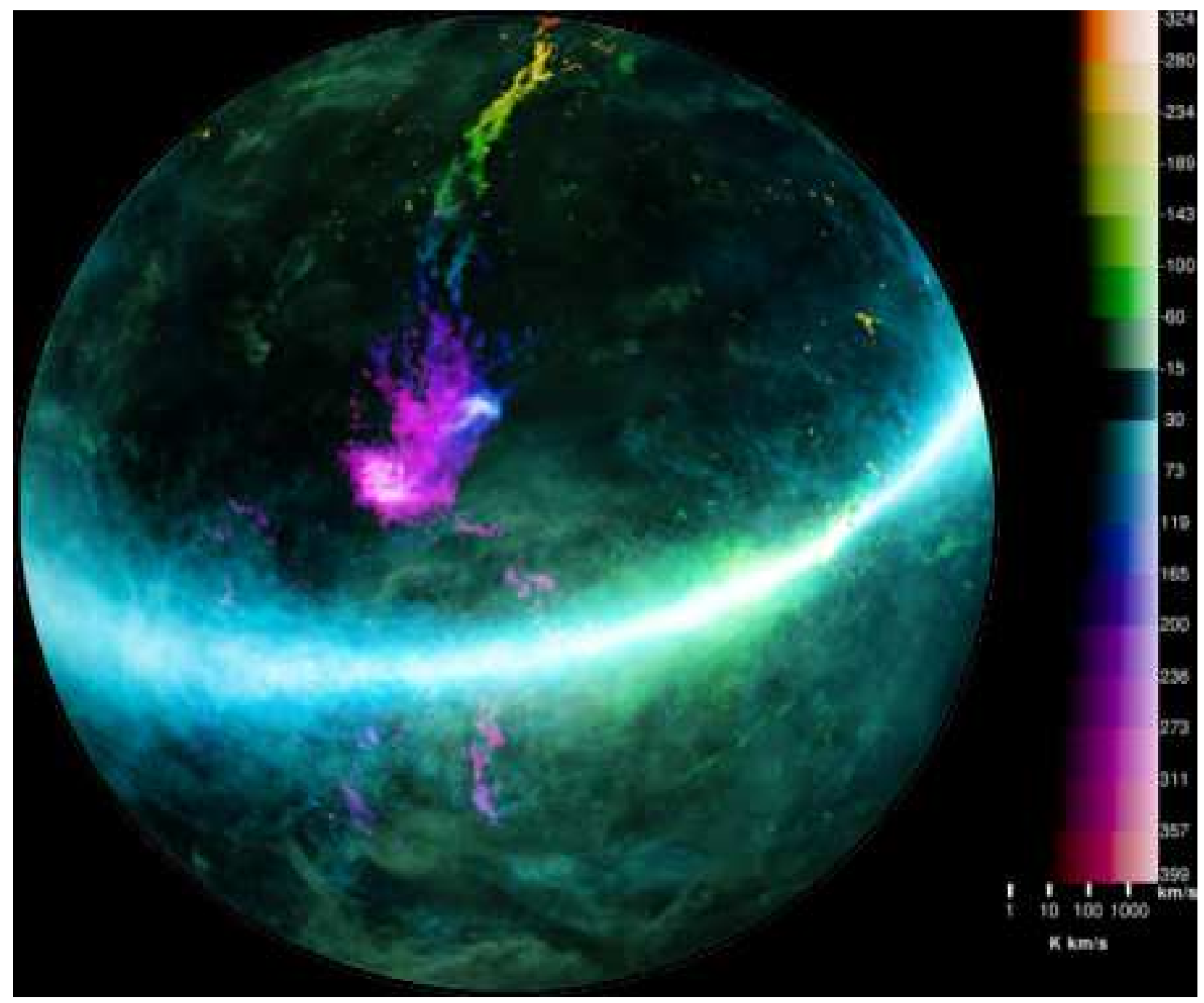

Fig. 4. - The entire GASS dataset shown in a ZEA projection centered on the south celestial pole with $0 \mathrm{hr}$ right ascension at the top and with RA increasing counter-clockwise. The colors correspond to integrations over velocity chunks of $\sim 40 \mathrm{~km} \mathrm{~s}^{-1}$ as indicated by the bar on the right of the image. The intensity of each color corresponds to the brightness temperature integrated over the $\sim 40 \mathrm{~km} \mathrm{~s}^{-1}$ velocity chunk, and is scaled logarithmically as shown by the horizontal extent of the color bar. Where emission exists at two different velocities, the intensities are combined using the "screen" algorithm in GIMP as described by Rector et al. (2007). Some artifacts from scanning were masked by hand. This image was made following the procedure detailed by Rector et al. (2007). 


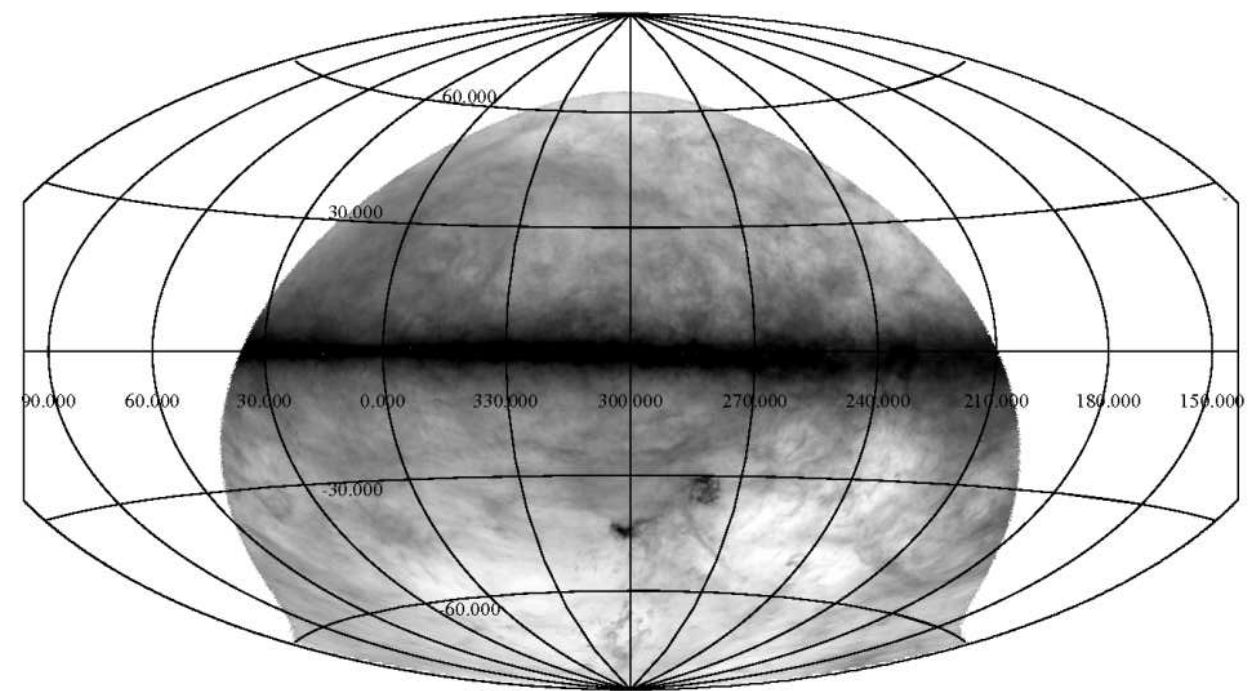

Fig. 5.- Total column density image in units of $10^{21} \mathrm{~cm}^{-2}$. The image is on an Aitoff projection in Galactic coordinates centered at $l=300^{\circ}$. The greyscale is logarithmic and shown in the wedge at the bottom. 


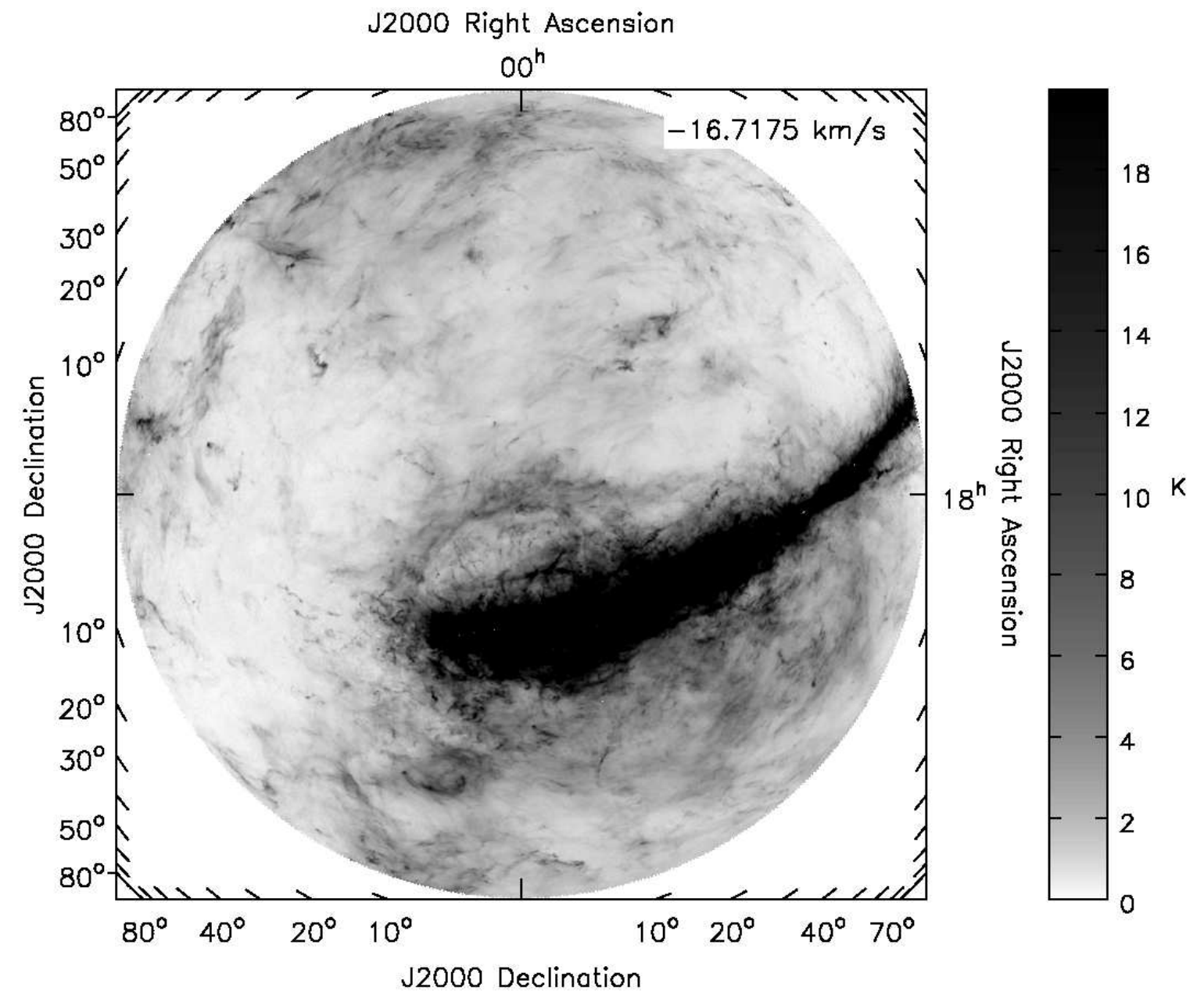

Fig. 6. - GASS image at $V_{L S R}=-16.7 \mathrm{~km} \mathrm{~s}^{-1}$. The greyscale goes from 0 to $20 \mathrm{~K}$ with a scaling power of -1 as shown in the wedge at the right. 


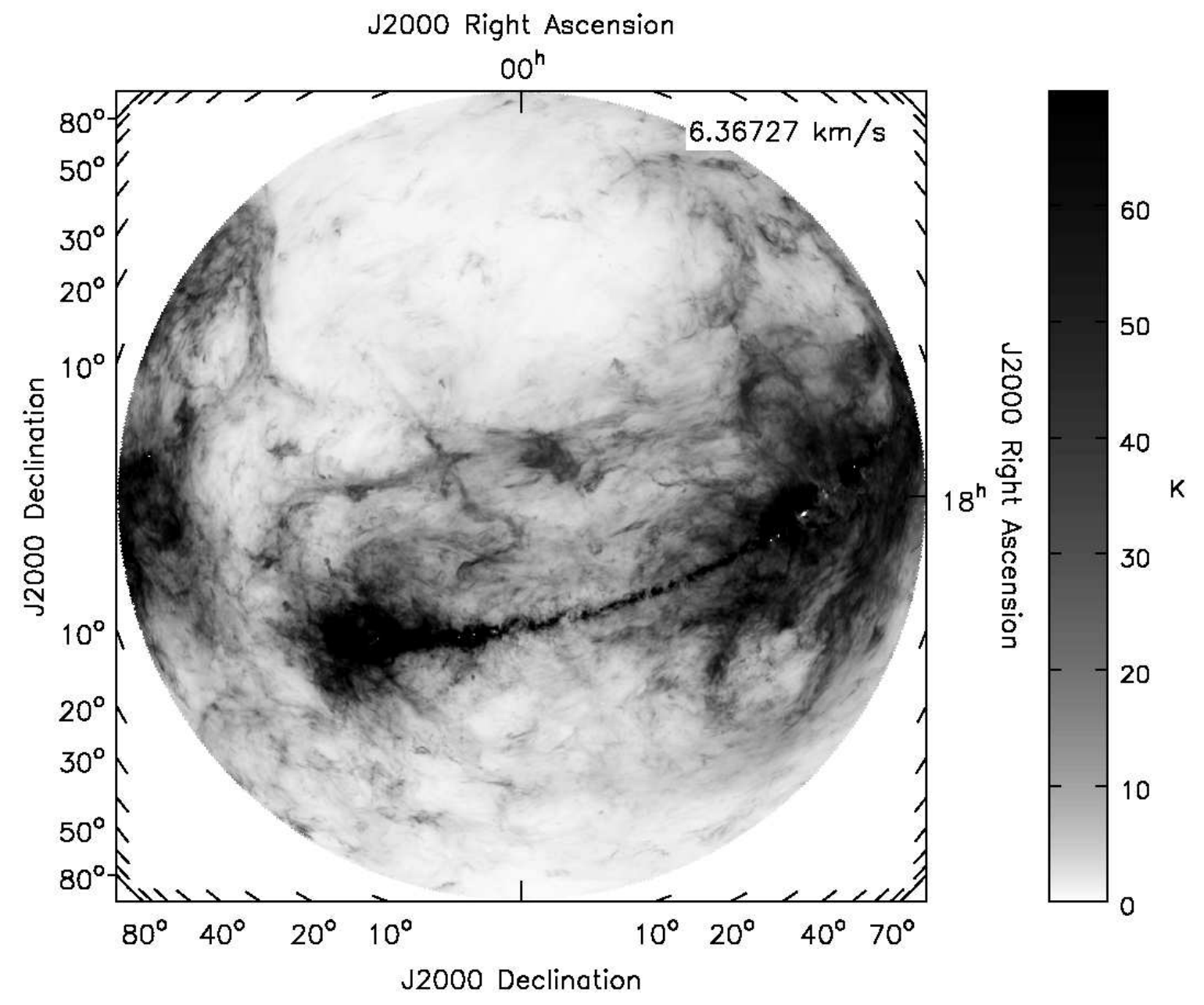

Fig. 7.- GASS image at $V_{L S R}=6.36 \mathrm{~km} \mathrm{~s}^{-1}$. The greyscale goes from 0 to $70 \mathrm{~K}$ with a scaling power of -1 as shown in the wedge at the right. 


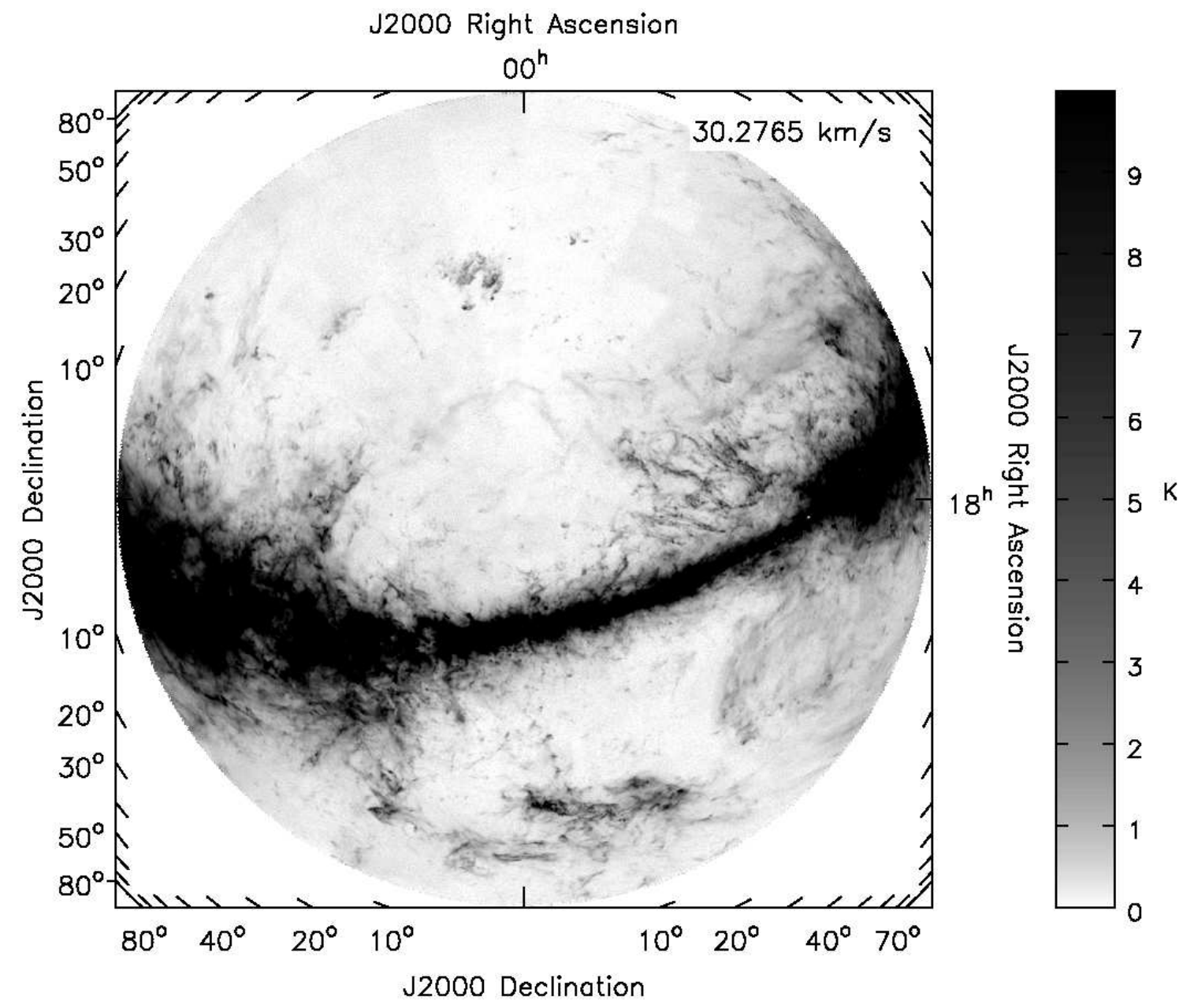

Fig. 8.- GASS image at $V_{L S R}=30.3 \mathrm{~km} \mathrm{~s}^{-1}$. The greyscale goes from 0 to $10 \mathrm{~K}$ with a scaling power of -1 as shown in the wedge at the right. The patchiness in the low level emission, particularly near RAs of $0 \mathrm{~h}$, is evidence of the difference in the stray radiation contribution to spatial areas observed in different observing epochs. 

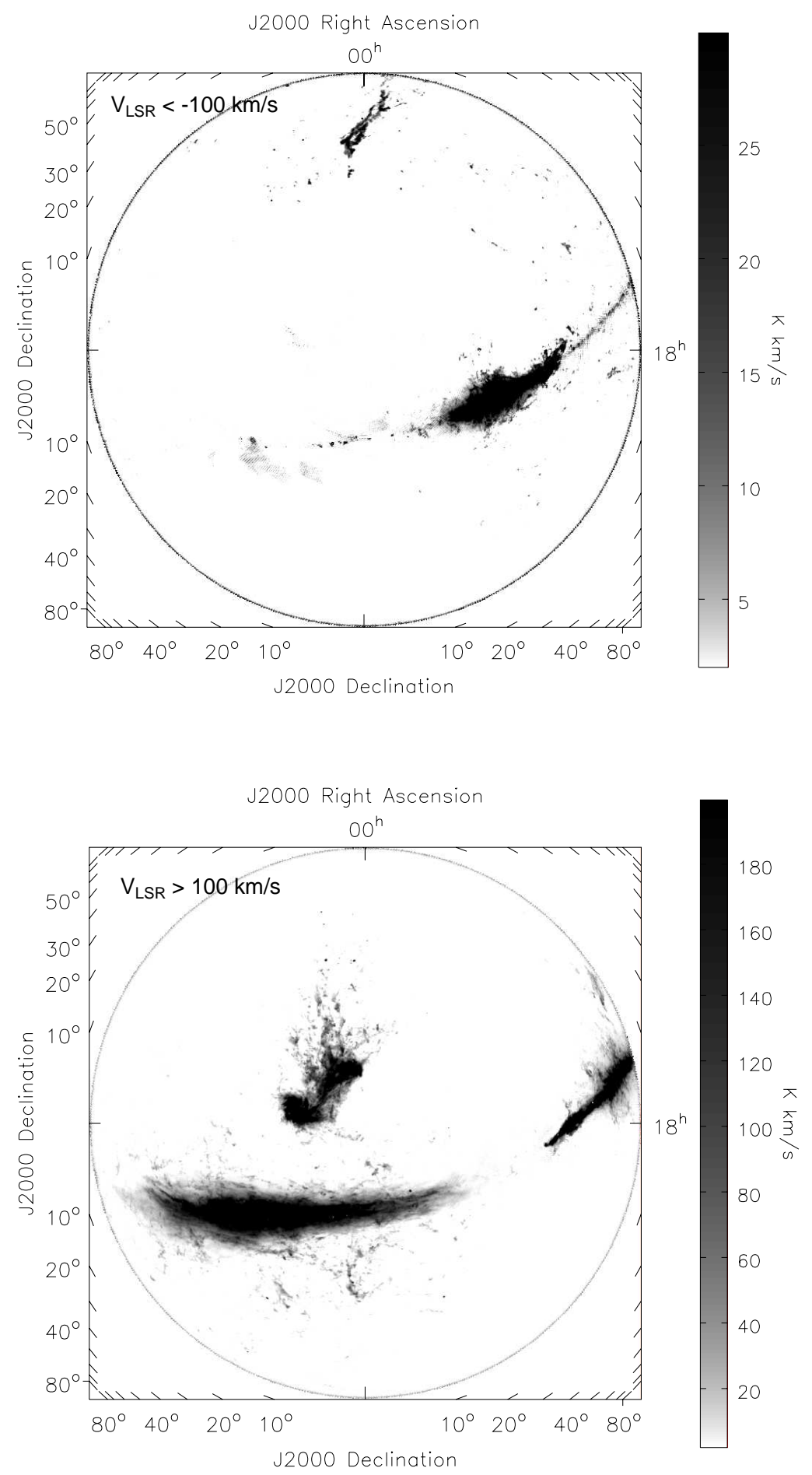

Fig. 9.- GASS high velocity sky for negative (top: $V_{L S R} \leq-100 \mathrm{~km} \mathrm{~s}^{-1}$ ) and positive (bottom: $V_{L S R} \geq 100 \mathrm{~km} \mathrm{~s}^{-1}$ ) velocities. The greyscales use a scaling power of -1 and are shown in the accompanying wedges. The negative high velocities are dominated by Galactic emission near to the Galactic center and a portion of the Magellanic Stream. The positive velocities are dominated by Galactic plane emission and the Large and Small Magellanic Clouds. Small HVCs are visible in both images. 

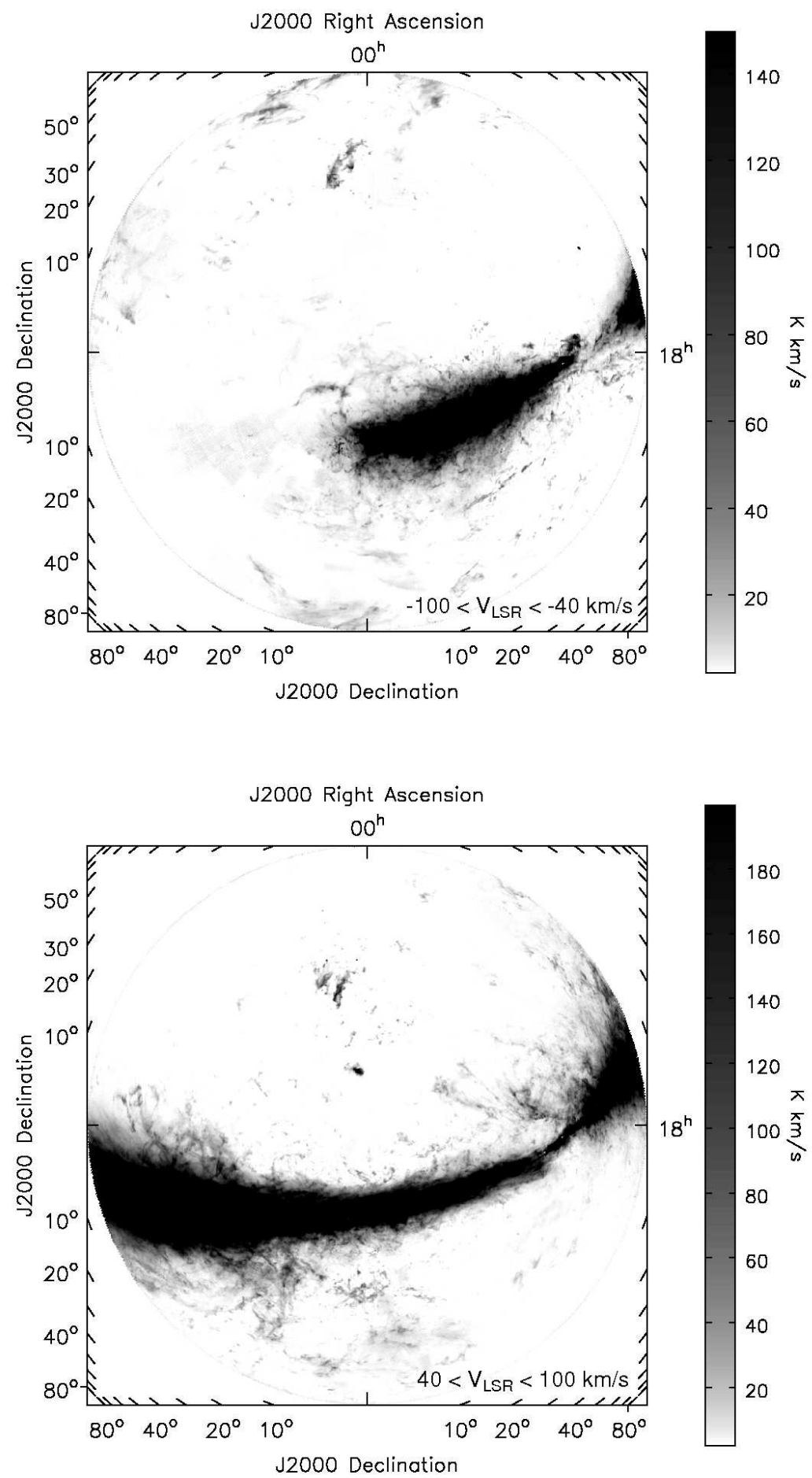

Fig. 10. - GASS intermediate velocity sky for negative (top: $-100<V_{L S R}<-40 \mathrm{~km} \mathrm{~s}^{-1}$ ) and positive (bottom: $40<V_{L S R}<100 \mathrm{~km} \mathrm{~s}^{-1}$ ) velocities. The greyscales use a scaling power of -1 and are shown in the accompanying wedges. A significant portion of the intermediate velocity sky is dominated by Galactic emission, however the filamentary nature of the extensions off the Galactic plane are noteworthy. 


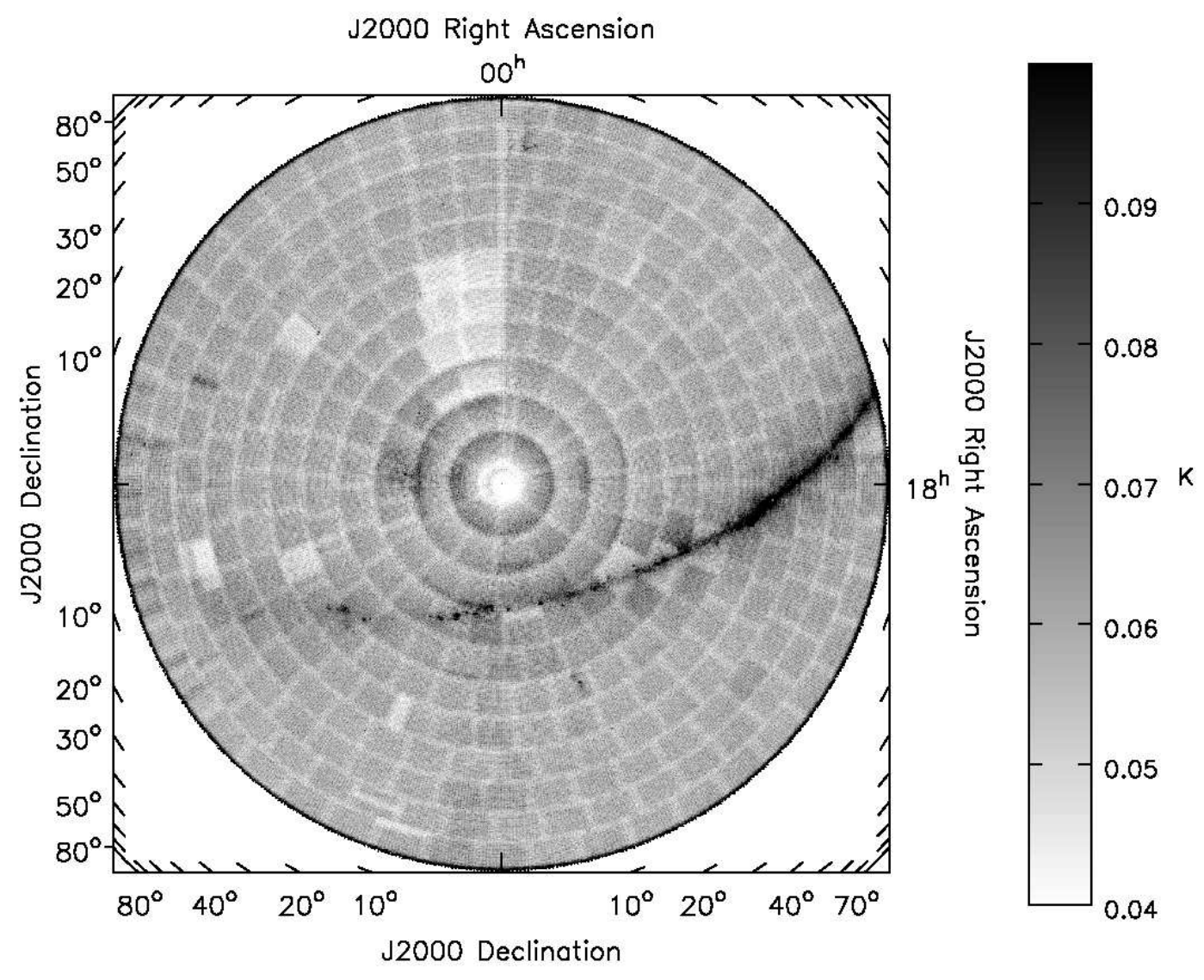

Fig. 11. - RMS across the GASS field calculated as the median of the rms in nine different blocks of $\sim 21$ emission-free channels. The greyscale is displayed in the color wedge to the right. The mode of the rms is $57 \mathrm{mK}$ across the field, with higher values in the Galactic Plane, towards Cen A and towards the Magellanic Clouds. 

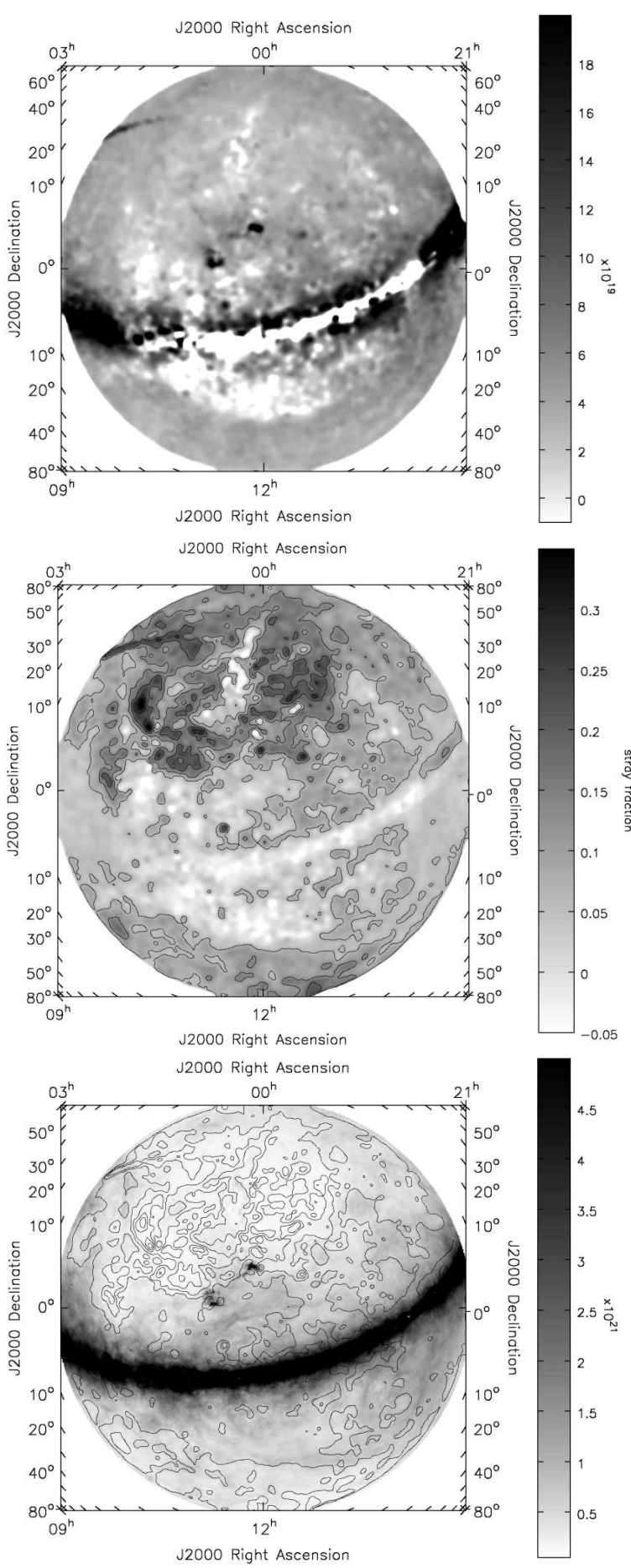

Fig. 12.- (top): The amount of stray radiation in GASS expressed as an equivalent $N_{H I}$. The greyscale is linear from $(-1$ to 20$) \times 10^{19} \mathrm{~cm}^{-2}$ with a scaling power of -0.5 . The large negative and positive values towards the Galactic Plane are due to baseline problems and not stray radiation. (middle): Fraction of total column density estimated to be due to stray radiation. The greyscale is linear from -0.05 to 0.35 as shown in the wedge. The contours run from -3 to $5 \times 0.065$. (bottom): Total column density of GASS. The greyscale goes from $5 \times 10^{19}$ to $5 \times 10^{21} \mathrm{~cm}^{-2}$ with a scaling power of 0.5. The contours are the same as in the middle panel. The linear feature in the top left is due to an artifact from regridding the LAB data. All of the stray radiation data have been smoothed to $2^{\circ}$ resolution. 


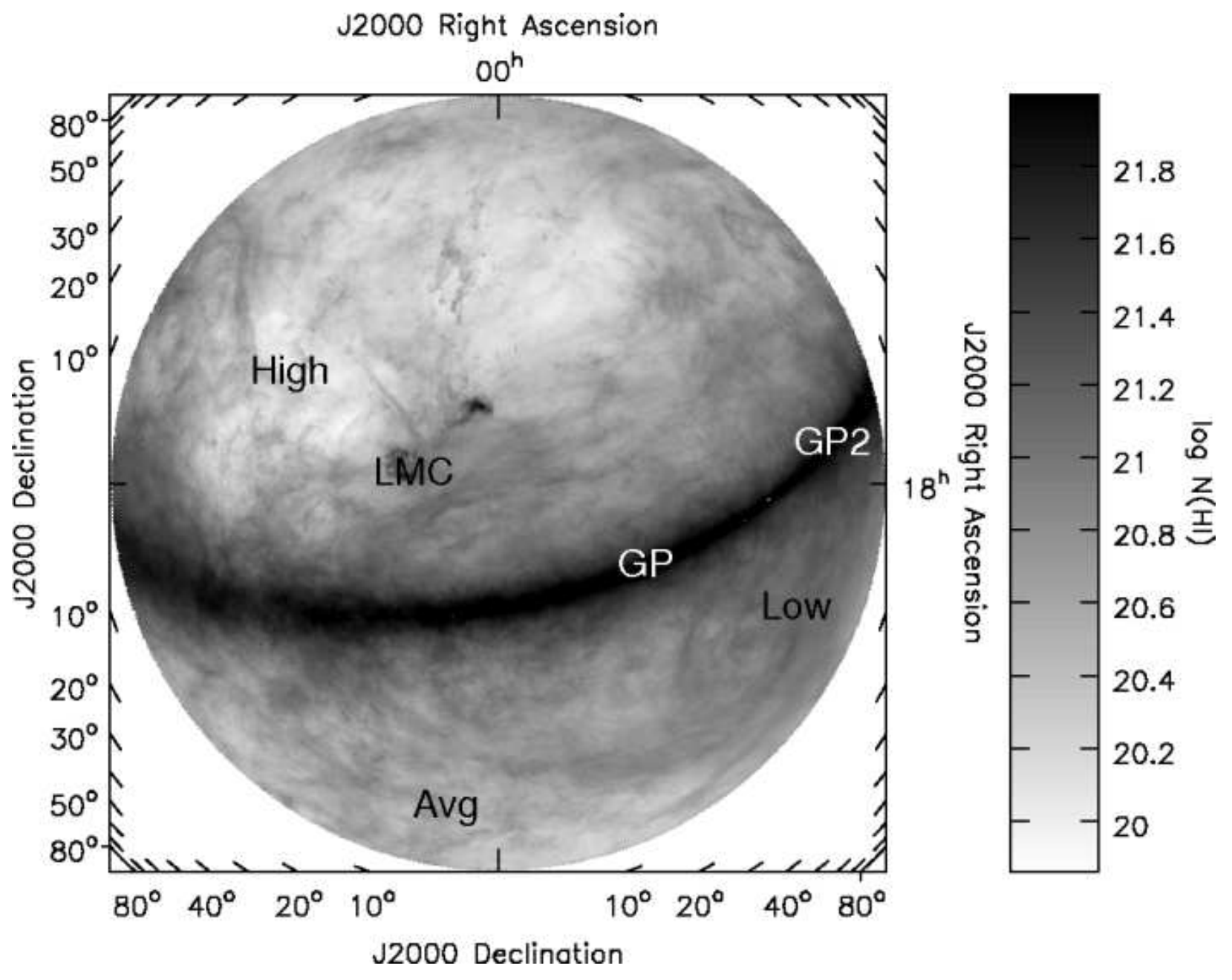

Fig. 13. - Annotated total column density image for GASS with the areas of low, average and high stray fraction used in Figure 14 marked together with the Galactic Plane and LMC areas referred to in Figure 16. The greyscale is $\log N_{H I}$ from 19.8605 to 22 in units of $\mathrm{cm}^{-2}$. 

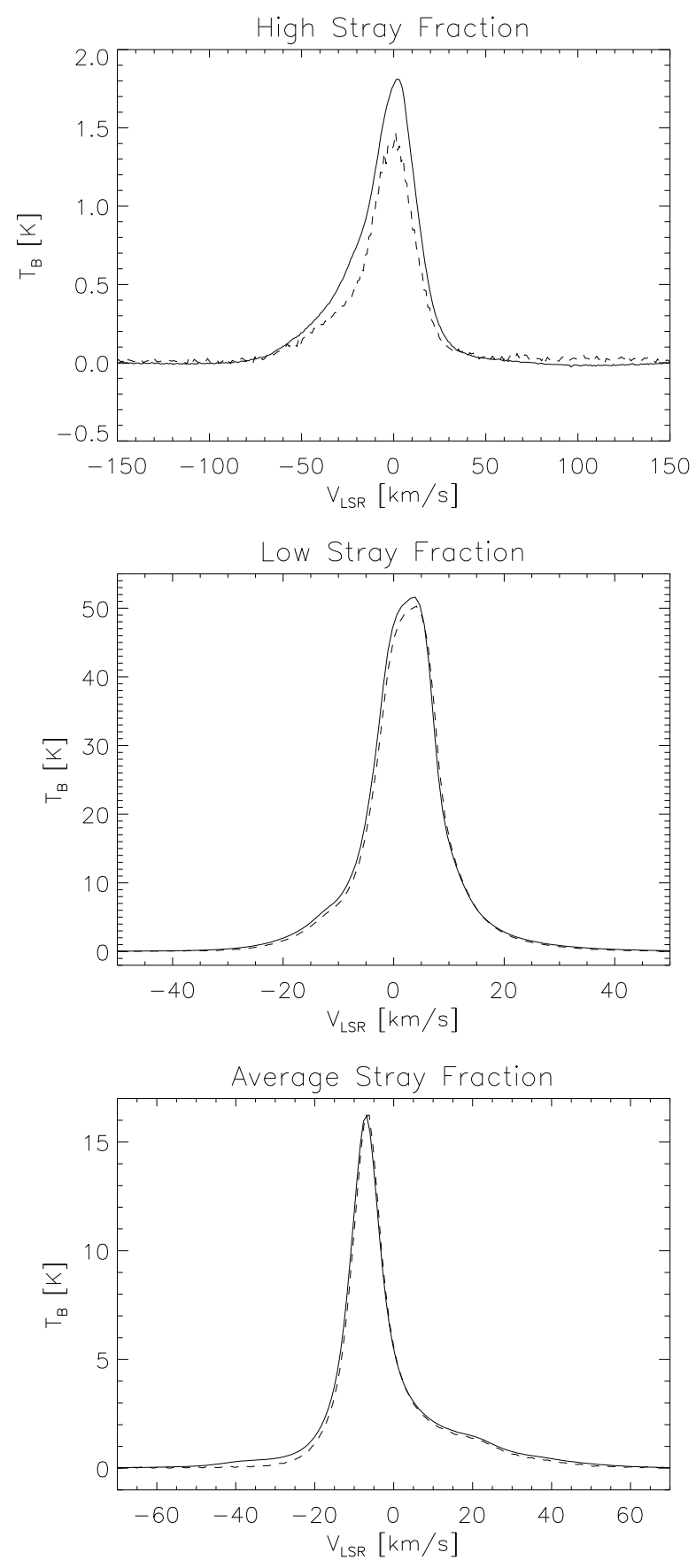

Fig. 14. - Comparison of spectra from GASS (solid line) and LAB (dashed line) towards the areas marked in Figure 13. These spectra, smoothed over $5^{\circ} \times 5^{\circ}$ region, show the effects of stray radiation, which produces slightly higher spectra in GASS than LAB and line wings. 

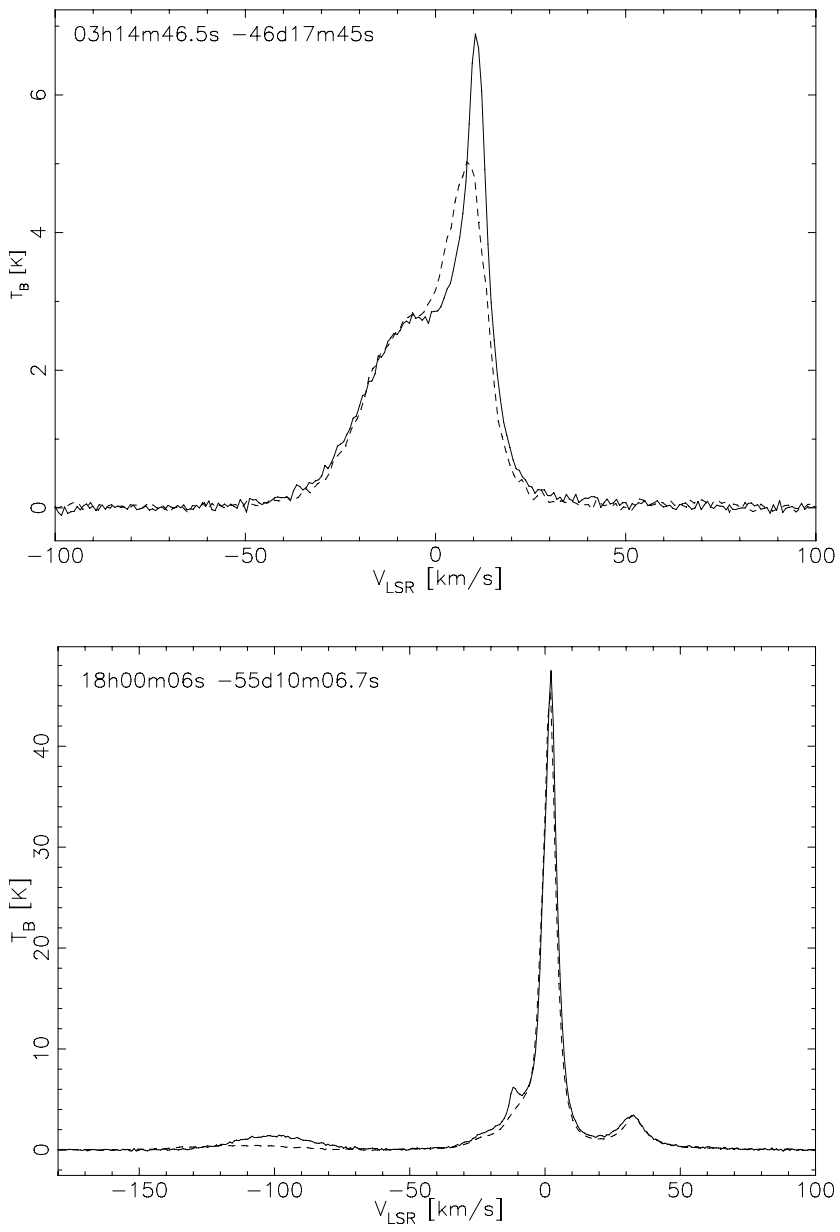

Fig. 15.- Comparison of spectra from GASS (solid line) and LAB (dashed line) at the full GASS resolution towards two regions. The general baseline quality of GASS data is good and the higher angular resolution GASS spectra show features that are not apparent in LAB. 

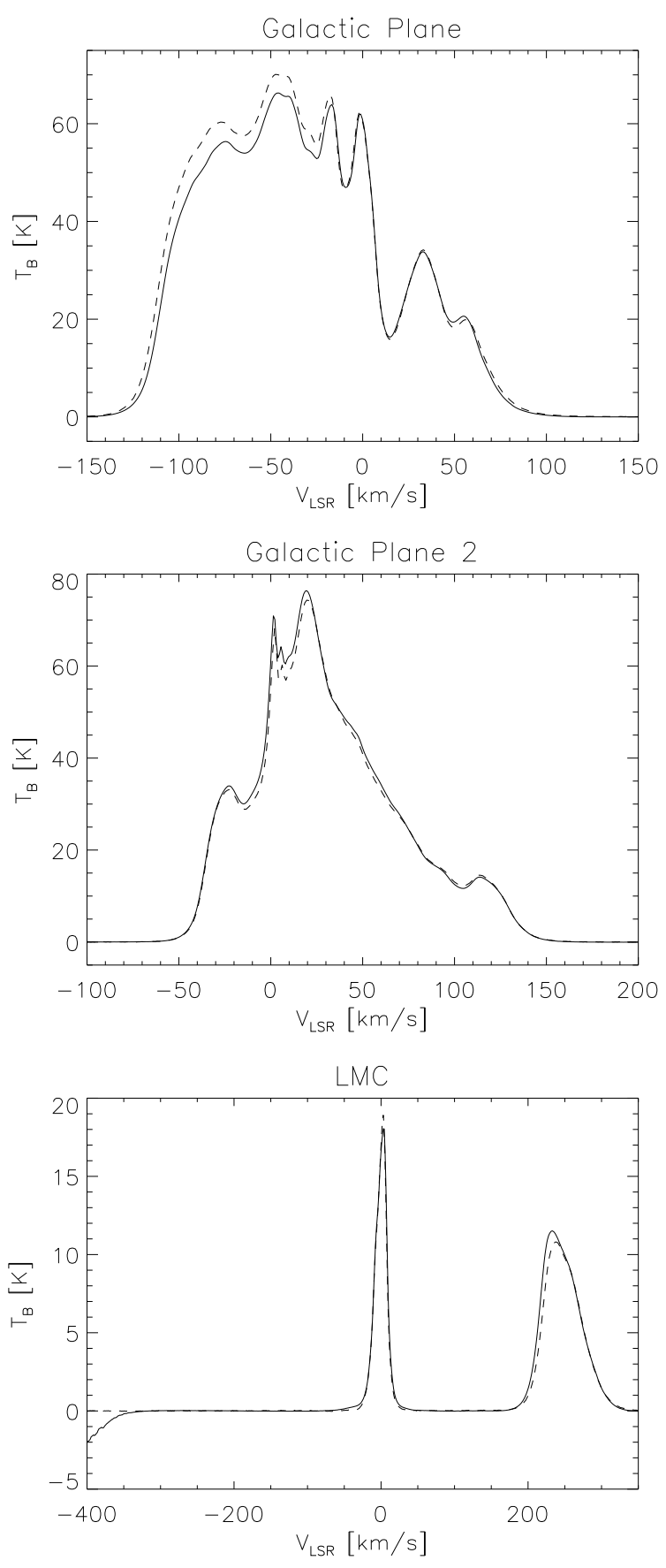

Fig. 16. - Comparison of spectra from GASS (solid line) and LAB (dashed line) towards the areas marked in Figure 13 as GP (top), GP2 (middle), and LMC (bottom). The spectra at the top and in the middle show the limitations in GASS bandpass solutions in the Galactic plane, which result in spectral distortions relative to LAB. The bottom spectrum shows the negative image at $V_{L S R}=-400 \mathrm{~km} \mathrm{~s}^{-1}$ from the LMC (located at $V_{L S R}=250 \mathrm{~km} \mathrm{~s}^{-1}$ ) caused by in-band frequency-switching. These spectra are smoothed over a $5^{\circ} \times 5^{\circ}$ region. 